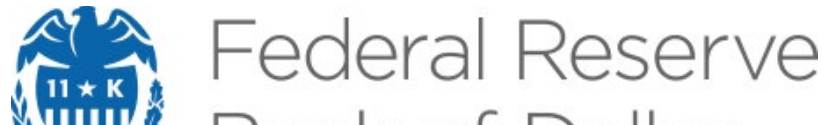 Bank of Dallas
}

\section{Rationally Inattentive Savers and Monetary Policy Changes: A Laboratory Experiment}

Andrea Civelli, Cary Deck and Antonella Tutino

\section{Working Paper 1915}

Research Department

https://doi.org/10.24149/wp1915

Working papers from the Federal Reserve Bank of Dallas are preliminary drafts circulated for professional comment. The views in this paper are those of the authors and do not necessarily reflect the views of the Federal Reserve Bank of Dallas or the Federal Reserve System. Any errors or omissions are the responsibility of the authors. 


\title{
Rationally Inattentive Savers and Monetary Policy Changes: A Laboratory Experiment ${ }^{*}$
}

\author{
Andrea Civelli ${ }^{\dagger}$, Cary Deck ${ }^{\ddagger}$ and Antonella Tutino ${ }^{\S}$
}

December 2019

\begin{abstract}
We present a model where rationally inattentive agents decide how much to save while imperfectly tracking interest rate changes. Suitable assumptions on agents' preferences and interest rate distribution allow us to derive testable theoretical predictions and their implications for monetary policy. We probe these predictions using a laboratory experiment with induced inattention that closely reflects the theoretical assumptions. We find that, empirically, the laboratory data corroborates the results of the theoretical model. In particular, we show that experimental subjects respond to changes in the interest rate policy environment with: (1) a decrease in savings when the utility gain from savings does not compensate for the cognitive cost of tracking the interest rate; (2) more informed and deliberate consumption/investment choices when the monetary authority stabilizes the economy by lowering the volatility of the policy rate, implementing a version of Delphic forward guidance; (3) a slight decrease in information processing but no behavioral changes in consumption when the monetary authority signals current monetary policy stance, implementing a version of Odyssean forward guidance; (4) a sizable decrease in investment when their perception of the outlook deteriorates. These experimental and theoretical findings agree with the empirical literature on the effect of monetary policy on households' consumption behavior in U.S. data and abroad.
\end{abstract}

Keywords: Rational Inattention, Experimental Evidence, Information Processing Capacity, Consumption

JEL Classification: C91, D11, D8, E20

\footnotetext{
* The views in this paper are those of the authors and do not necessarily reflect the views of the Federal Reserve Bank of Dallas or the Federal Reserve System.

${ }^{\dagger}$ Andrea Civelli, University of Arkansas, Walton College of Business, Department of Economics, Business Building 402, Fayetteville, AR 72701, andrea.civelli@gmail.com.

${ }^{\ddagger}$ Cary Deck, University of Alabama and Chapman University, cdeck@cba.ua.edu.

§Antonella Tutino, Federal Reserve Bank of Dallas, tutino.antonella@gmail.com.
} 


\section{Introduction}

Economists and policy makers are concerned about how changes in monetary policy and communication of those changes may affect the private sector's reactions in terms of consumption and investment. In standard rational expectations models, agents optimally and quickly react to changes in the economic environment, no matter how small or smooth the changes in the policy instruments or policy statements are. Thus, under the rational expectation framework, monetary policy is seen as a continuous balancing act between policy changes and information released about changes and forecasts of future changes.

However, private agents' consumption and investment responses to the same policy changes vary greatly according to whether people shift their attention to the monetary policy environment. For instance, in periods of relative stability, agents may delay or not respond at all to fluctuations that are small and/or unimportant to them, regardless of how much or how little information is released. By contrast, in turbulent times, more of their attention may be devoted to tracking changes in the monetary policy environment leading to more erratic reactions in their behavioral response of consumption and investment while agents try to parse information about economic conditions.

This paper accounts for these behavioral patterns by modeling agents as having limited cognitive ability to process information about the monetary policy environment, i.e., they are rationally inattentive in the sense of Sims (2006) and Sims (2003). In contrast with the rational expectations paradigm, the rational inattention structure is less concerned about how much information should or should not be released, but whether and when the information released elicits strong behavioral reactions. We hence propose a model where rationally inattentive agents face monetary policy changes, and we derive testable implications of consumption and investment choices in response to those changes. These implications are tested using a controlled laboratory experiment.

Sims pioneered the theory of rational inattention by postulating that economic agents optimally choose the amount of information for a given decision problem. The cost of processing information is based on Shannon's mutual information between prior and posterior beliefs. Following this approach, Tutino (2013) explicitly considers the cost implied by Shannon's mutual information as a cognitive cost. This cost captures the limits of people to map quickly and precisely all the available information about the economic environment into behavioral choices. Moreover, Tutino (2013) shows that for a given shadow cost of processing information, agents react to exogenous economic changes by varying the informativeness of the signals they require to select consumption choices. This concept, known as elastic 
information processing capacity, implies that different economic stimuli correspond to different behaviors, according to whether the decision-maker deems it beneficial to save processing effort by accepting lower consumption or she prefers to incur the cost of paying additional attention to increase the informativeness of signals and make sharper consumption choices.

Building on that literature, this paper proposes a simple 2-period model where in the first period an agent receives an endowment and decides how to allocate it between consumption and savings. The return on savings is given by the prevailing interest rate. The agent is rationally inattentive and has limited cognitive capacity to process information about the interest rate. Thus, the decision problem of the agent amounts to choosing how much information she wants to process about the interest rate functional to her choice of consumption in period 1 and investment in the risky asset (savings).

We postulate that agents have quadratic utility. This assumption implies a subdued response to changes in the interest rate as they have limited impact on agents' lifetime utility. While invoked mainly for analytical tractability, this assumption is backed by the empirical findings of, e.g., Bachmann et al. (2015) and Roth and Wohlfart (2019) that show muted behavioral responses of households to changes in monetary policy. With quadratic utility, and for given distributional assumptions for the interest rate, we can characterize the solution of the model and derive a set of theoretical predictions to be taken to the laboratory.

To translate the theoretical problem into a laboratory setting, we provide the subjects with a fixed endowment to gamble with in a set of lotteries. The outcome of the lottery (per-period payoff) depends on the subjects' selection of which lottery to play together with the particular draw on the return of the gamble in each lottery. Each of these lotteries represents a different lifetime utility from the theoretical model and takes on the values from the quadratic utility specification. Different values of the payoffs for each lottery chosen by the subjects encode the uncertainty about which interest rate would occur when they decide consumption and savings. Hence, choosing a lottery is equivalent to choosing savings with uncertain rate of return in the model. The initial endowment is constant in both the model and the experimental settings.

In the model and in the experiment we postulate a uniform distribution from which interest rates (returns) are drawn. Subjects know the distribution of investment returns but they do not know the realized outcome. However, they can acquire signals to reduce uncertainty about which return has been drawn before selecting their lottery. We provide the participants with an array of signals with varying levels of accuracy. The signals provide additional information by narrowing the range from which a particular return was drawn. 
The reduction in uncertainty a subject can achieve is linked to the cognitive effort the subject exerts to reveal the signal information. The higher the precision of the signal, the narrower the intervals of possible profitability it provides. However, the cognitive effort to extract the information content of the signal increases with signal precision.

We capture cognitive efforts by requiring participants to solve logic puzzles, with more difficult puzzles demanding more effort to be solved by the subjects. Given that Rational Inattention theory is based on cognitive effort rather than time spent going through a different number of tasks, logical puzzles are suitable to gauge how hard subjects think, as shown in Civelli and Deck (2018). We relate the difficulty of the puzzles to the informativeness of the signals with harder puzzles corresponding to more precise information about which return has been drawn. The difficulty of the puzzles range from trivial (corresponding to an uninformative signal) to very hard (corresponding to a perfectly revealing signal). Upon completion of the tasks, participants select their desired lottery based on the updated (informed) prior. Once the outcome of the lottery is revealed, participants gain their perperiod payoff and move on to the next period. Participants are paid on the basis of their accumulated payoffs over the course of the experiment. Other than the opportunity cost of processing information versus playing more lotteries, there is no time limit on any given period. By repeatedly exposing the subjects to each decision problem, we can collect data on their behavior toward consumption and savings as well as information/signal choices. These data are used to assess whether the prediction of the model are verified.

We show that experimental subjects' choices are generally consistent with the rational inattentive representative agent behavior. More importantly, we formally compare the experimental outcomes to the rationally inattentive model and a model that has full information. We find that subjects' behavior is better captured by the rational inattention model: participants behave as limited in their information processing capacity rather than possessing full information and reacting quickly and precisely to changes in the economic environment as postulated by rational expectations models with infinite processing capacity.

We focus on four main predictions from our theoretical model and design experimental treatments directly conducive to verifying whether laboratory evidence corroborates these predictions.

The first prediction we test concerns the value of information. The theory predicts that when the utility gain of processing more information does not provide a sufficient compensation for the cognitive effort of precisely tracking the interest rate, the agent prefers to increase consumption in the first period and reduce savings. This precautionary consumption 
result of the model stems from the following rationale. With a stochastic interest rate, the reduction in uncertainty about the return requires a cognitive effort that is higher than the benefit, captured by the curvature of the utility function. With quadratic utility, the gains from better information are not compensated by the cognitive cost of learning more about changes in the interest rate. As a result, the safe choice for the participant is to consume most of their endowment in period 1 and acquire little information about the interest rate. We find that the subjects in the experiment prefer to exercise low effort and increase consumption in the first period rather than invest in the risky return on savings, a behavior which is consistent with the theoretical trade off between gains from information acquisition and required cognitive efforts. This is true even if, on average, a more informed subject receives a higher utility reward. This result has a stark monetary policy implication: if people's utility functions make deviations of interest rates policy unimportant to them, interest rate changes are unlikely to elicit behavioral reactions. Moreover, both the theoretical and the experimental findings corroborate the empirical evidence in U.S. and international data of a relatively small impact of monetary policy on households' decisions (as pointed out by, e.g., Roth and Wohlfart, 2019).

The second and third predictions of the model concern the effectiveness of forward guidance in affecting agents' behavioral choices. Following Campbell et al. (2012), we identify two forms of forward guidance: Delphic and Odyssean forward guidance. ${ }^{1}$ A form of Delphic forward guidance can be thought of a reduction in the volatility of the interest rate. ${ }^{2}$ Implementing this policy in our framework, the model finds that decreasing the volatility of the policy rate reduces agents' cognitive cost of processing information for any precision chosen. As a result, agents are able to make more informed consumption and investment decisions when monetary policy is conducted with this form of Delphic forward guidance. In particular, the model predicts that, with less volatile interest rate, the lower cognitive cost associated to tracking the interest rate leads consumption/investment responses to changes in the monetary policy stance that are systematic and deliberate. Empirically, the subjects' behavior in the laboratory is consistent with this prediction.

As a result, both theoretically and in the laboratory there is a material improvements in welfare (evaluated as the difference between full information and realized outcomes, as

\footnotetext{
${ }^{1}$ As described in Campbell et al. (2012), Delphic forward guidance refers to communication of the central bank about the outlook, whereas Odyssean forward guidance refers to a commitment of the central bank to keep rates stable, see Eggertsson and Woodford (2003).

${ }^{2}$ This type of forward guidance would naturally emerge as the solution for the policy rate based on an optimal control problem with quadratic loss function as in Taylor (1993)
} 
a percentage of full information) when this form of forward guidance is adopted. Rational Inattentive representative agent as well as experimental subjects enjoy higher utility by making more deliberate and precise consumption and investment choices when faced with a less volatile interest rate. Moreover, a less volatile interest rate lowers the cognitive burden of tracking changes in the interest rate for the agents, contributing to the increase in the economy-wide welfare.

The second form of forward guidance - and the third theoretical prediction of the model - concerns Odyssean forward guidance. This type of forward guidance corresponds to a situation in which the central bank announces whether the current monetary policy stance is loose or tight and commits to the announcement. The version of the Odyssean forward guidance we employ in the paper is one in which the monetary authority signals whether the interest rate in the current period is low or high and the signal is accurate with $90 \%$ probability. In principle, this increase in predictability of the moentary policy stance makes it easier for the private sector to track movements of the policy rate.

The model predicts that increase in predictability is associated with a modest decrease in attention. More importantly, the model predicts that this version of Odyssean forward guidance has no effect on agents' behavior with respect to a situation in which such a policy is not adopted. The lack of effectiveness in influencing behavior stems from the fact that once the agents have optimally selected their signal, they choose their consumption conditional on the information acquired, disregarding the central bank's announcement. Since the signal acquired is generally not more informative than the one selected without central bank's commitment, agents' behavioral choices are not affected by this policy. We find that the laboratory subjects behave in accordance with the theory, with negligible differences in consumption outcomes depending on whether or not the announcement is made. As a result, there is no material improvement in welfare stemming from this policy.

These theoretical and experimental results on the efficacy of Delphic and Odyssean forward guidance are strongly supported by the empirical literature. ${ }^{3}$ To our knowledge, this is the first paper to provide theoretical and laboratory evidence that agree with the findings on these two forms of forward guidance applied to U.S. and cross-country data on aggregate consumption and investment.

The fourth theoretical prediction is that a deterioration in the public perception of the outlook dampens investment (i.e., increases consumption in the first period) for a given

\footnotetext{
${ }^{3}$ See, e.g., Andrade and Ferroni (2018), McKay et al. (2016), Campbell et al. (2019) and Ehrmann et al. (2019)
} 
information precision. In the model, this prediction is obtained by postulating a decrease in agents' time discount factor. This lower degree of patience makes today's consumption more valuable to the agents than investment. This drop can be interpreted as an increase in impatience of the private sector that may occur after a prolonged recovery from a recession. Since savings is less appealing from the private sector's perspective, agents are less interested in processing information about the interest rate applied to investment. This theoretical finding agrees with the empirical findings of, e.g., Roth and Wohlfart (2019), Bachmann et al. (2015) and, more recently Yagan (2019). We find that the optimal consumption path from the theoretical model under rational inattention closely captures participants' consumption and investments choices in the laboratory experiment. The implication of this finding is that the influence of monetary policy on private sector's behavior not only depends on the size of the change in the policy instrument, but also on the timing of the change along the business cycle.

The rest of the paper is organized as follows. After discussing related literature in the second part of this section, Section 2 formally presents the theoretical decision problem under rational inattention. It discusses the properties of the problem's solution and lays out the testable predictions. Section 3 introduces the experimental setting and the treatments implemented to verify the theoretical predictions. A mapping between experimental setup and the theoretical model is formally established. Section 4 shows the congruence of the experimental with the theoretical predictions. Finally, Section 5 offers some concluding remarks. Details and Instructions of the experiment are in the Appendix.

\section{$1.1 \quad$ Related Literature}

This paper relates to two broadly defined areas of research. The first strand concerns the importance of cognitive limits on economic decisions. While there is an extensive literature documenting attention limits in economics, ${ }^{4}$ there has been significantly less research on testing models of limited attention in general and rational inattention in particular in an experimental setting. Among the papers that empirically test attention limits, Caplin and Dean (2011) tests a sequential search models where agents face a large number of alternatives. The key difference between their paper and ours is that our subjects choose higher information precision by solving progressively more difficult tasks so that the number of alternatives is tightly linked to the cognitive effort. Other papers study models of

\footnotetext{
${ }^{4}$ See, e.g., in the context of consumer choices: Chetty et al. (2009),Allcott and Taubinsky (2015), Santos et al. $(2012)$
} 
information acquisition and their impact on choices in strategic settings when agents are not fully rational. In the experimental literature, Gabaix et al. (2006), Khaw et al. (2016) and Goecke et al. (2013) study dynamic models of information acquisition without assuming that subjects are rational. These papers focus mostly on information acquisition while our paper is centered around information processing, explicitly connecting the acquisition of information to the cognitive cost of mapping that information into behavioral choices.

The set of papers that are the closer to our paper (Pinkofskiy (2009), Cheremukhin et al. (2015), Dean and Neligh (2017) and Civelli et al. (2018)) all use Shannon's entropy costs as the basis of their experimental analysis. Cheremukhin et al. (2015) exposes subjects to gambles in the lab to estimate the cost of information processing as well as attitude to risk. Dean and Neligh (2017) empirically tests the validity of rational inattention models under different specifications of the information cost based on Shannon's mutual information. Civelli et al. (2018) is the closest to our paper as it employs cognitive tasks to explicitly map information acquisition into consumption choices of risk averse subjects. In contrast with the previous literature, in this paper, we abstract from considering risk attitude and tests of alternative specifications of the rational inattention models. Instead, we propose a model where monetary policy changes are the primary stimuli to the economic environment that trigger changes in information processing. We encode the complexity of deciphering monetary policy changes for our experimental subjects by associating progressively more demanding cognitive tasks in order to obtain sharper information. In this context, we focus on how rationally inattentive subjects react to monetary policy variations and measure their information processing and consumption choices.

The second broad strand of the literature this paper relates to is on information frictions and the signaling channel of monetary policy. ${ }^{5}$ In particular, a number of recent papers empirically document the interaction between information rigidities, inflation expectations and monetary policy. Evidence based on surveys of firms (Kumar et al. (2015), Coibion et al. (2019)) show mixed response of the effect of monetary policy on economic outcomes. With respect to households' expectations, Easaw et al. (2013), Roth and Wohlfart (2019), Bachmann et al. (2015), Binder (2017) and Khaw et al. (2017) shows changes in information acquisition that relate to changes in behavioral choices.

In this paper, we track subjects' information acquisition and processing directly together with consumption choices. This way we are able to disentangle whether a particular change

\footnotetext{
${ }^{5}$ See e.g., Baeriswyl and Cornand (2010) and Melosi (2016) for examples of how interest rates serve as communication tools from monetary policy to firms.
} 
in monetary policy trigger changes in information acquisition and consumption behavior.

Finally, the paper also relates to the recent debate on the relative effectiveness of Delphic vs. Odyssean forward guidance of monetary policy. ${ }^{6}$ McKay et al. (2016) and Campbell et al. (2019) both warn on the limits of forward guidance in affecting private sector's behavior, with Delphic forward guidance more effective then Odyssean forward guidance in nudging behavior when private sector expectations are not rational as in Eusepi and Preston (2018) and firms have rational inattention as in Gaballo (2016). ${ }^{7}$ This paper provide experimental support for the theoretical findings of these paper by showing that while a central bank signal on the monetary policy stance (a form of Odyssean forward guidance) is generally ineffective in influencing participants' behavior toward consumption and savings, having a less volatile interest rate as Delphic forward guidance tool could modify consumption choices.

\section{Theoretical Framework}

In this section we focus on the theoretical framework which lays out the optimization problem of the agent. Moreover, the model provides a structure for the experimental setting and delivers predictions that we test in the laboratory experiment. The model features a rationally inattentive agent that lives for two periods. At the beginning of period 1 , the agent receives a certain endowment of 1 unit of consumption good and one asset. She can transfer part of that endowment to period 2 by investing in the asset at the prevailing interest rate, $r$. The second period utility is discounted at the rate $\beta$. Let $c_{1}$ and $c_{2}$ denote consumption at period 1 and period 2 respectively, then for a given interest rate, consumption in period 2 is determined by the choice of consumption in period 1 as residual of the initial endowment:

$$
c_{2}=r\left(1-c_{1}\right) .
$$

We assume that the agent is rationally inattentive and she has limited information-processing capacity to process information about the interest rate. This means that, from the perspective of the agent, the interest rate payed by the asset in the second period is a random variable. Moreover, without knowledge of the interest rate, consumption in both period 1 and 2 are random variables since different realizations of the interest rate give rise to different

\footnotetext{
${ }^{6}$ As described in Campbell et al. (2012), Delphic forward guidance refers to communication of the central bank about the outlook, whereas Odyssean forward guidance refers to a commitment of the central bank to keep rates stable, see Eggertsson and Woodford (2003).

${ }^{7}$ Gaballo (2016) develops a Rational Inattention model with Delphic forward guidance in central banks may be successful in changing firms' pricing behavior.
} 
consumption options. Thus, the agent needs to decide how much information she wants to acquire about the interest rate functional to her consumption choices. Following Tutino (2013) and Civelli et al. (2018), we express the choice of information about interest rate useful for consumption as the joint distribution of consumption and the interest rate. Specifically, the agent starts out with a prior on interest rate that has distribution $g(r)$. She can reduce uncertainty about her prior by acquiring signals on the interest rate as they relate to her consumption possibilities in period 1 which, implicitly, also define how much endowment she transfers to period 2 consumption. This choice variable is represented by the joint probability $p\left(c_{1}, r\right)$. Given the joint probability of consumption and the interest rate, the signals can be interpreted as conditional probability of interest rate given consumption in period 1 as derived by Bayes' rule:

$$
p\left(r \mid c_{1}\right)=\frac{p\left(c_{1}, r\right)}{\int p\left(c_{1}, r\right) d r}
$$

The optimal choice of the joint distribution $p^{*}\left(c_{1}, r\right)$ depends on the information processing capacity of the agent. We postulate that the maximum amount of information that she can extract from consumption about the interest rate is limited by the Shannon's channel capacity. Before processing any information, the uncertainty about interest rate can be expressed by the entropy of the prior on $r, \mathcal{H}(r) \equiv-\mathbb{E}\left[\log _{2}(g(r))\right]$. Since consumption and interest rate are related in the agent's decision, knowledge of consumption in period 1 provides information about interest rate. Thus the reduction of uncertainty about the interest rate that can be achieved through knowledge of consumption can be expressed as conditional entropy of interest rate given consumption, $\mathcal{H}\left(r \mid c_{1}\right)$. Shannon's theory posits that the information flow between the random variables interest rate, $r$, and consumption in period $1, c_{1}$, expressed as $I\left(c_{1}, r\right)$, is bounded by a limit, $\kappa$, which represents the maximum amount of information that can be extracted from $c_{1}$ about $r$. In formulae:

$$
I\left(c_{1}, r\right)=\mathcal{H}(r)-\mathcal{H}\left(r \mid c_{1}\right) \leq \kappa
$$

The bound $\kappa$ is expressed as the number of bits of information that the agent can process about consumption and interest rate and poses a limit on the informational content of the signals that the agent can choose. Let $u\left(c_{i}\right)$ be the utility of the agent at periods $i=(1,2)$ with $u^{\prime}>0$ and $u^{\prime \prime}<0$, and let $\beta$ be the discount factor on period 2 consumption, $c_{2}$. Then, the optimization problem of the agent in period 1 is given by: 


$$
\begin{array}{ll}
\max _{p\left(r, c_{1}\right)} & \mathbb{E}_{1}\left\{\int\left[u\left(c_{1}\right)+\beta u\left(c_{2}\right)\right] p\left(c_{t}, w_{t}\right) \mu\left(d c_{1}, d r\right) \mid \mathcal{I}_{1}\right\} \\
\text { s.t. } & \kappa=I\left(p\left(c_{1}, r\right)\right) \\
& c_{2}=r\left(1-c_{1}\right) \\
& p\left(c_{1}, r\right) \in \mathcal{D}(w, c) \\
& g(r) \text { given }
\end{array}
$$

where $\mu(\cdot)$ is the Dirac measure that accounts for discreteness in the optimal choice of $p(c, r)$, while $\mathcal{D}(r, c)$ restricts the choice of the agent to be drawn from the set of distributions, $\mathbb{E}_{1}$ is the conditional expectation defined with respect to the $\sigma$-algebra generated by $\mathcal{I}_{1}$. As per usual, it is assumed that the discount factor $0<\beta<1$ and utility $u\left(c_{i}\right)$ for $i=1,2$ is twice continuously differentiable. The program can be solved by Lagrangian methods. Let $\theta$ indicate the Lagrange multiplier associated with constraint (2), which can be interpreted as the shadow cost of processing information. Then the total cost of choosing a signal of information content $\kappa$ is given by $\theta \kappa$. We postulate an environment with elastic capacity on agents' information-processing. That is, for a given marginal cost of processing information, $\theta$, the agent chooses the optimal amount of information conveyed by the signal as determined by the informational content of $p\left(c_{1}, r\right)$. The program in equations (1)-(5) is a well-posed mathematical problem, but with state and control variables that are infinite dimensional. Moreover, a discretization of this framework is also a well-posed problem and returns as a solution the equilibrium distribution $p^{*}\left(c_{1}, r\right)$ which, from Bayes' rule as well, can be represented as

$$
p^{*}\left(c_{1}, r\right)=p^{*}\left(c_{1} \mid r\right) g(r)
$$

where the marginal interest rate distribution is equal to the prior, $\int p^{*}\left(c_{1}, r\right) d c_{1}=g(r)$, to satisfy model's internal consistency. The conditional distribution $p^{*}\left(c_{1} \mid r\right)$ embeds the effects of more accurate information about interest rate provided by the selected signal to sharpen consumption choices. To build intuition on the character of the solution and derive testable predictions from the model, the next section specializes the framework in equations (1)-(5) with specific distributional assumption on the prior $g(r)$ which is directly conducive to the experimental setting, as well as a particular case with quadratic utility. 


\subsection{A useful closed-form solution}

In order to bring the model closer to the experimental setting and study the solution properties of the model to be tested in the laboratory, we assume that the prior on the interest rate $g(r)$ is uniformly distributed over the interval $R=[\underline{r}, \bar{r}]$, that is $g(r) \backsim U(R)$, where $\underline{r}<\beta^{-1}<\bar{r}$. Moreover, to facilitate the implementation of the experimental design, we assume that the signals on interest rates available to the agents partition the interest rate space into sub-intervals of the same length, proportional to the precision of the signal. The distribution implied by the signals is also piece-wise uniform within each partition.

Let $R_{i}$ indicate a generic partition of $R$ determined by the signal of precision $i$, such that $R_{i}=\left[r_{i}, \bar{r}_{i}\right]$. Then, the solution of the problem in terms of consumption can be compactly written as:

$$
\beta^{-1} u^{\prime}\left(c_{1}\right)=\mathbb{E}_{R_{i}}\left\{r u^{\prime}\left(r\left(1-c_{1}\right)\right)\right\}
$$

where $u^{\prime}(\cdot)$ is the derivative of utility with respect to consumption in period $1, c_{1}$, and the expectation $\mathbb{E}_{R_{i}}$ is taken with respect to the information provided by the optimal signal $p^{*}\left(r \mid c_{1}\right)$ for the interval $R_{i}$.

To further characterize the solution, let us assume that the utility $u(c)$ takes on the form:

$$
u(c)=\left\{\begin{array}{cl}
\alpha(c-\bar{c})^{2} & c<\bar{c} \\
0 & c \geq \bar{c}
\end{array}, \bar{c} \gg \bar{r}\right\}
$$

which postulates that the satiation point $\bar{c}$ is much higher than the maximum amount that could be consumed by saving all the endowment in the first period even in the case of the highest possible realization of interest rate, $r=\bar{r}$.

Given the definition of the expectation operator $\mathbb{E}_{R_{i}}$ and of the corresponding variance $\mathbb{V}_{R_{i}}$, the rationally inattentive consumption choice for the case of quadratic utility and uniform, piece-wise linear interest rate signals can be written as:

$$
c_{1}=\frac{\bar{c}+\beta\left(\mathbb{E}_{R_{i}}\left(r^{2}\right)-\mathbb{E}_{R_{i}}(r) \bar{c}\right)}{1+\beta \mathbb{E}_{R_{i}}\left(r^{2}\right)}=\frac{\bar{c}+\beta\left(\mathbb{E}_{R_{i}}^{2}(r)+\mathbb{V}_{R_{i}}(r)-\mathbb{E}_{R_{i}}(r) \bar{c}\right)}{1+\beta\left(\mathbb{E}_{R_{i}}^{2}(r)+\mathbb{V}_{R_{i}}(r)\right)}
$$

Compared to the reference result in the full information problem, $c_{1}=\frac{\left(\bar{c}+\beta\left(r^{2}-r \bar{c}\right)\right)}{1+\beta r^{2}}$, we see that, in (7), the certain value of the interest rate $r$ is replaced by its expected value within the interval revealed by the signal. We can use equation (7) to further characterize the testable prediction of the model and to guide our laboratory experiment. 


\subsection{Testable predictions}

The validity of the closed-form solution in eq. (7) relies on the functional form of the utility in eq. (2.1). This specification generally implies a relatively flat slope of the utility. The economic interpretation of this assumption is that agents typically devote limited cognitive bandwidth to monetary policy news in choosing consumption decisions that increase their utility. We invoke this assumption that leads to a closed-form solution by trading off a more prominent role of information processing choices for clearer and more direct implications of the theory on consumption behavior.

Furthermore, the assumption on the slope of the utility is consistent with empirical evidence on households and firms. ${ }^{8}$ Under this assumption on the utility specification, the solution of the closed form model in equation (7) produces four theoretical predictions that can be directly tested in the laboratory experiment:

\subsubsection{Testable Prediction 1}

Consumption in period 1 decreases as the expected interest rate increases. However, as implied by eq. (7), the utility benefit of processing more information about the interest rate is bounded. The first part of the prediction follows immediately from $\frac{\partial c_{1}}{\partial \mathbb{E}_{R_{i}}(r)}<0$, while the second part of the prediction clearly stems from the functional form (2.1). Thus, the model predicts agents to generally acquire limited information and change their consumption infrequently.

\subsubsection{Testable Prediction 2}

Consumption in period 1 increases as the perceived volatility of the interest rate increases. This prediction stems from $\frac{\partial c_{1}}{\partial \mathbb{V}_{R_{i}}(r)}>0$. This theoretical result makes sense because in our model consumption in period $1, c_{1}$, is the "safe" asset when interest rates are perceived as a random variable. As a result, an increase in the volatility of the stochastic rate of return to savings (i.e., $\mathbb{E}_{R_{i}}$ ) makes agents more willing to consume now rather than save for an uncertain payoff tomorrow.

\footnotetext{
${ }^{8}$ See for firms, inter alia Coibion et al. (2019) and Kumar et al. (2015) and for households, inter alia, Roth and Wohlfart (2019) and Easaw et al. (2013). See Section 1.1 for more references.
} 


\subsubsection{Testable Prediction 3}

A decrease in the discount rate $\beta$, lowers consumption $c_{1}$ and makes information processing less valuable. The prediction stems from $\frac{\partial c_{1}}{\partial \beta}<0$. This theoretical result also makes sense. A decrease in the discount rate can be interpreted as a deterioration of the outlook from the agents' perspective. With less confidence in future earnings, agents retreat to the safety of $c_{1}$. The decision of consuming more today also implies that there is less even less incentive in processing information about tomorrow's return on savings as expressed by the interest rate.

\subsubsection{Testable Prediction 4}

Enhanced predictability of the interest rate path does not affect consumption behavior. This theoretical results stems from the fact that rationally inattentive agents have optimally chosen their signal on the interest rate taking into account their information-processing capacity as well as their utility. As a result, a public signal that contains less information than what is optimally chosen would be mostly disregarded as agents rely on their own, richer, information set to learn about the interest rate. Likewise, a public signal that contains more information than what they would have optimally chosen would be processed with noise: had the agents had sufficient information processing capacity and/or more interest in processing information as commanded by their utility, they would have already optimally chosen a more informative signal about the interest rate. Thus, a commitment from the monetary authority to provide an accurate path of the monetary policy stance would have limited material impact on consumption.

\section{Experimental Design and Implementation}

This section lays out details of our within subject experimental design including a description of the tasks designed to implement the model in Section 2 as well as the four treatments.

\subsection{Experimental Task}

The basic idea of the experiment is to create a decision problem that in which one decides how much to consume and how much to save when the interest rate is uncertain. To simplify the task, we present the decision to subjects as choosing one among several lotteries where each lottery has a well specified payoff for each of the equally likely possible states of the 
world that could be realized. Specifically, subjects select among 11 possible lotteries and there are 32 distinct possible states of the world. Each lottery corresponds to a choice of period 1 consumption. The state of the world corresponds to a possible interest rate. Because period one consumption and the interest rate determine period 2 consumption per equation (4), this is enough to fully determine the discounted expected utility, which gives the payoff for a given lottery in a given state (see equation 1). In the experiment, the payoffs are described as prizes, the lottery choice is described as selecting a prize card (from 1-11), and the realized state is described as drawing a prize number (from 1-32). The basic process of making a consumption/savings choice and learning the realized payoff constitutes what we refer to as a period.

The prize number (interest rate) in a period is drawn before a subject make her prize card (consumption/savings) choice, but this realizations is not reveled to the subject. Subjects only know the distribution of potential outcomes. However, a subject can acquire signals to reduce uncertainty about which prize number has been drawn before selecting a prize card. We provide subjects an array of signals with varying levels of accuracy. A signal gives additional information about the realized state that has been drawn by identifying a narrower range of possible prize numbers. How much reduction in uncertainty a subject can achieve is linked to the cognitive effort the subjects exerts in order to reveal the information content of the signals. The higher the precision of the signal, the narrower the intervals of possible prizes it provides, but the more significant the cognitive effort a subject must exert to extract signals' information becomes. We impose this increasing cognitive effort for more precise signals by giving subjects a fixed number of progressively more difficult logic puzzles to solve. More details about signals, intervals, and cognitive tasks are discussed in Section 3.2. With the introduction of these signals, the decision problem of the subjects becomes how much information they want to acquire before selecting a prize card (savings rate).

Both the representative agent from Section 2 and the experimental subjects have a prior on the interest rate, $g(r)$, that is a discrete uniform distribution across the possible realizations of $r$. The support of the distribution encompassing all the possible outcomes for $r$ is known. Later we provide the specific parameter values used in the experiment for the different variables discussed in Section 2. Also, in accordance with the model, the structure of the signals belongs to the uniform family. The informativeness of the signal is captured in the model with the capacity of the Shannon channel, $\kappa$ in equation (2). In the experiment, we interpret $\kappa$ as quantity of information for a given shadow cost of processing information, denoted by $\theta$. Our use of incrementally more difficult logic puzzles to receive more precise 
information aligns with shadow costs being related to the cognitive effort required to process information.

The interval of possible prizes (interest rates) revealed to the subject after acquiring a signal defines the corresponding optimal conditional distribution in the theoretical framework, $p^{*}\left(c_{1} \mid r\right)$. By narrowing the support of the possible draws, subjects can modify their prior $g(r)$ with a more concentrated distribution reflecting lower uncertainty about a lotteries' return. For instance, in our base set-up subjects that choose to process information with level 1 precision would go from 32 possible prizes to 16, scaling back by half their payoff uncertainty. The observed realization of a particular payoff is a draw from the optimal distribution $p^{*}\left(c_{1} \mid r\right)$. 


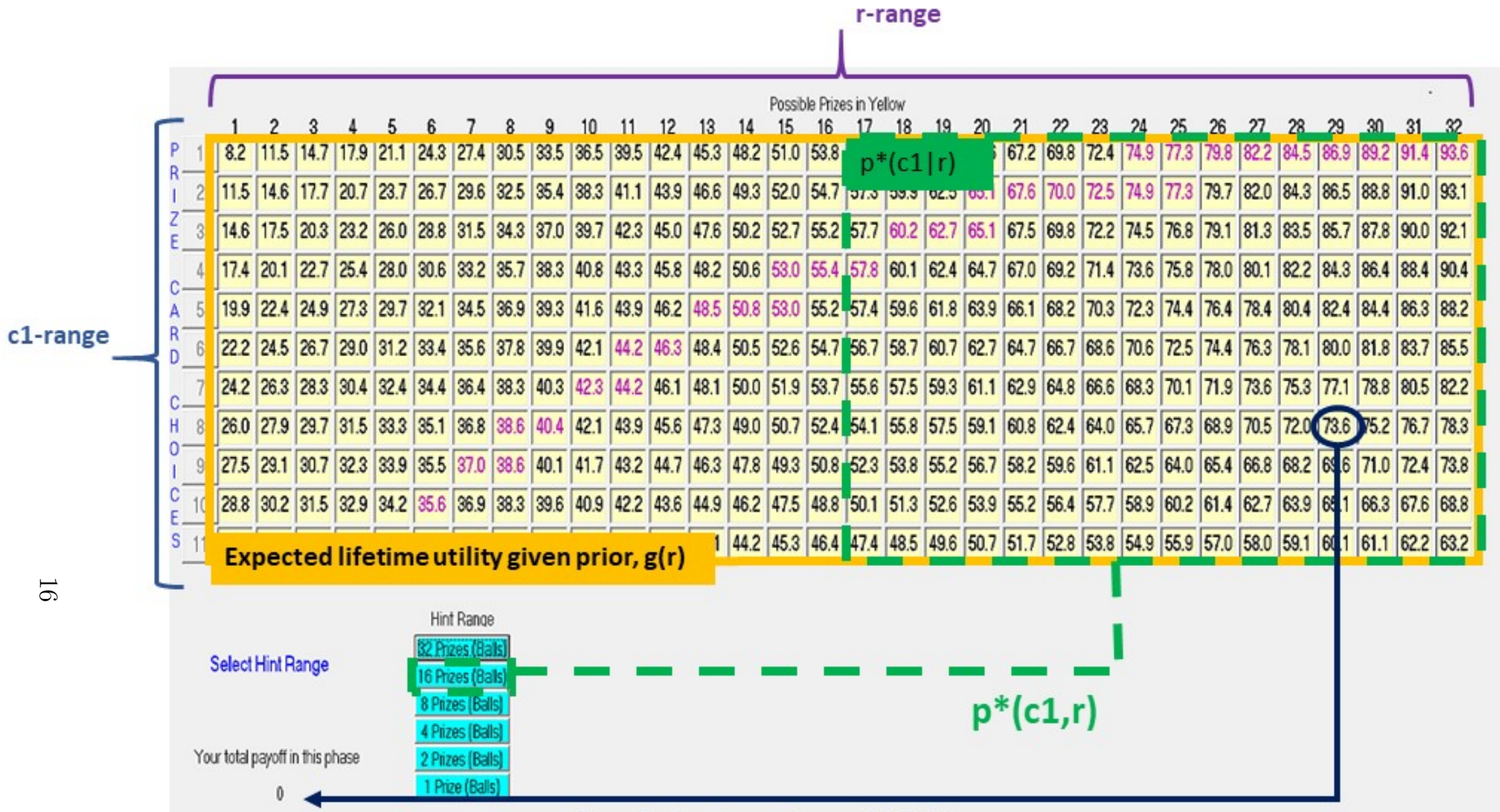

Cumulative draws from $p^{*}(\mathrm{c} 1 \mid \mathrm{r})$

Figure 1: Mapping from the theory to the experiment: A screenshot of the experimental interface with elements of the theoretical framework superimposed. The matrix of lottery payoffs in the yellow box are a function of the $r$ drawn in the column (32 possibilities) and the consumption choice of the subject in the row (11 options). Red payoffs indicate the full information optimum for a given $r$. Subjects select a signal by clicking on one of the cyan buttons on the SW corner of the interface, which reveals a subset of columns containing the true realization of $r$ (the green dashed box, which corresponds to theoretical conditional distribution of consumption $\left.p^{*}\left(c_{1} \mid r\right)\right)$. In the example, the second signal precision is selected and the right side of the $r$ support is revealed. Signal selection and restricted support jointly correspond to the choice of the optimal distribution $p^{*}\left(c_{1}, r\right)$. 
The experiment is deployed in the laboratory using the interface shown in Figure 1. The possible prizes - the yellow cells in the table - follow the quadratic surface of the lifetime utility in Section 2. The possible realized states (prize numbers), which correspond to the range of $r$ in the model, are drawn from a uniform distribution of 32 potential states of the world and are represented by the columns of the table in Figure 1. These states of the world encode the stochasticity of interest rate in $g(r)$ uniformly distributed over the interval $R=[1,32]$ from Section 2.1. The rows of the table denote the 11 possible choices of $c_{1}$ that the subject can make.

Signal selection in the experiment is done by clicking one of the cyan boxes in the lower left corner of the figure. For instance, let us hypothetically consider a selection of level 1 signal precision. This is the case shown in Figure 1 as well. Acquisition of the signal would reduce the number of possible outcomes and hence payoffs (the expected lifetime utility given the prior $g(r))$ by half, and either side of the payoff matrix could be revealed as containing the true realization of $r$. In the figure, this case is illustrated by going from the full space enclosed by the yellow, solid-line box to the reduced space enclosed by the green dashedline box on the right side of the matrix. ${ }^{9}$ Once the signal reveals that only prizes 17-32 are possible, the green box corresponds to the theoretical optimal conditional distribution $p^{*}\left(c_{1} \mid r\right)$.

Signal selection together with the restricted support the signal affords correspond in the model to choosing the optimal amount of information about interest rate movements functional to the choice of current consumption. ${ }^{10}$ In the model, this is exactly the choice of selecting the optimal joint distribution $p^{*}\left(c_{1}, r\right)$. Once the signal is revealed, the subjects select a row $\left(c_{1}\right)$ in the restricted support bounded by the green box. This row is then highlighted in blue. ${ }^{11}$ This is equivalent to drawing a particular realization of $c_{1}$ from the optimal distribution conditional on the signal, $p^{*}\left(c_{1}, r\right)$. After the interest rate is revealed, the row selection together with the revelation of which state of the world has occurred (a particular column in the table), the payoff for the period is realized, highlighted in blue, and added to cumulative payoff displayed in the lower left corner of the screen. This would complete a decision period. In our example, if row 8 is chosen and column 29 is drawn, the subject would earn a payoff of 73.6. Finally, note that in Figure 1 the payoffs in red font provide the full information benchmark of the optimal $c_{1}$ associated with each possible

\footnotetext{
${ }^{9}$ In the laboratory, the computer would dynamically gray out the left-hand side of the payoff table leaving only the space in the green box in yellow.

${ }^{10} \mathrm{Or}$, equivalently to the saving choice.

${ }^{11}$ In the experiment, the subject must confirm the row choice before the outcome is revealed.
} 


\begin{tabular}{|c|c|c|c|}
\hline Precision & \multicolumn{2}{|c|}{ Intervals Description } & $\begin{array}{l}\text { Information } \\
\text { Flow (bits) }\end{array}$ \\
\hline 0 & 1 interval of length 32 : & {$\left[\begin{array}{ll}1 & 32\end{array}\right]$} & 0 \\
\hline 1 & 2 intervals of length 16 : & {$\left[\begin{array}{ll}1 & 16\end{array}\right]\left[\begin{array}{ll}17 & 32\end{array}\right]$} & 0.3 \\
\hline 2 & 4 intervals of length 8: & {$\left[\begin{array}{ll}1 & 8\end{array}\right] \ldots\left[\begin{array}{ll}24 & 32\end{array}\right]$} & 0.6 \\
\hline 3 & 8 intervals of length $4:$ & . $\left[\begin{array}{ll}28 & 32\end{array}\right]$ & 0.9 \\
\hline 4 & 16 intervals of length 2 : & {$\left[\begin{array}{ll}1 & 2\end{array}\right] \ldots\left[\begin{array}{ll}30 & 32\end{array}\right]$} & 1.2 \\
\hline 5 & 32 intervals of length 1 : & {$[1] \ldots[32]$} & 1.5 \\
\hline
\end{tabular}

Table 1: Signal structure: precision levels, signal characteristics, and information flows of the signals.

interest rates.

\subsection{Signal characteristics and cognitive tasks}

In the model, we postulate that the representative agent is limited in her ability to process information about interest rate variation. In the experiment, we encode this limit through the structure of the precision level of the signals. This section describes the characteristics and information content of these signals.

Subjects have several levels of signal precision available, ranging from uninformative to fully information. For a fixed shadow cost of processing information, $\theta$, we measure the informativeness of the signal, $\kappa$, as amount of information (in bits) contained in the signal. In the experimental setting, we fix the number of tasks that the subject is asked to solve to obtain an uncertainty reduction. We chose to assign the same number of tasks for each informative signal precision to emphasize the importance of cognitive effort required to solve the task over the time spent on an individual task.

The amount of information in each signal is linked to the cognitive difficulty of the logic puzzles the subject is asked to solve. Signals with higher precision require solving progressively more difficult puzzles. As summarized in Table 1, subjects have access to 6 levels of signal precision. The levels start from a basic Level 0 signal, which is uninformative and simply replicates the prior of the distribution of outcomes, to a Level 5 signal, which perfectly reveals the prize draw. Letting $j$ indicate the precision level, a signal identifies one of the $2^{j}$ intervals containing $2^{5-j}$ draws into which the full support of draws is partitioned. We can compute the information flow of the signals by finding their variation in entropy starting from the uniform prior $g(r)$. The information flows of the signals are reported in 


\begin{tabular}{|c|c|c|}
\hline $\begin{array}{l}\text { Precision } \\
\text { Level }\end{array}$ & Logic Puzzle Attributes & $\begin{array}{l}\% \text { Task } \\
\text { Correct }\end{array}$ \\
\hline 0 & 1 puzzle: level 0 & $0.99 \%$ \\
\hline 1 & 2 puzzles: level 1 and level 1 & $0.80 \%$ \\
\hline 2 & 2 puzzles: level 1 and level 2 & $0.65 \%$ \\
\hline 3 & 2 puzzles: level 2 and level 2 & $0.50 \%$ \\
\hline 4 & 2 puzzles: level 3 and level 3 & $0.35 \%$ \\
\hline 5 & 2 puzzles: level 4 and level 5 & $0.20 \%$ \\
\hline
\end{tabular}

Table 2: Signal structure: precision levels, logic puzzle attributes and expected success rate of cognitive tasks. Note: a level k puzzle has $\mathrm{k}$ attribute changes.

the last column of Table 1. Because the signal structure is such that signals pertain to uniform distributions and the supports of the signals are proportional, our signals have the property that the change in entropy is constant from one precision level to the next, and equal to 0.3 bits. This structure means that, once a signal is revealed, the expectation $\mathbb{E}_{R_{i}}$ and the corresponding variance $\mathbb{V}_{R_{i}}$ in the optimal choice of which lottery to play described in equation (7) in Secion 2.1 are taken with respect to the updated prior on the states of the world as it emerges from the signal.

The actual logic puzzles are based on a visual task developed by Civelli and Deck (2018). These puzzles take on the form of a $(3 \times 3)$ graphical matrix in which eight images are provided and one is omitted. Subjects must identify the missing image among a set of alternatives, after analyzing the patterns of attributes shared by the eight images in the matrix. The puzzles are similar to the well known Raven's Progressive Matrices, and their level of difficulty is correlated with the reasoning abilities of an individual. ${ }^{12}$ An example puzzle is illustrated in Figure 2, while the corresponding solution set with eight images is shown in Figure 3.

Each image in the puzzle has six attributes that could change: shape, size, shade of the filling, orientation, border style, and corner marker style. These attributes are allowed to change following six schemes of patterns: orthogonal - along rows and columns; diagonal along main or counter-diagonal; and corners - from NW to SE and from SW to NE. The difficulty level of a matrix is determined by the number of attributes that varies. In the example image, only one attribute varies - shape varies along the counter-diagonal; thus the correct solution is option "D" in Figure 3. Given the puzzle calibration exercises conducted

\footnotetext{
${ }^{12}$ We opt to use these puzzles rather than Raven's Progressive Matrices because the level of difficulty can be controlled and there are potentially a very large number of puzzles of any given difficulty level. These puzzles are also free.
} 
by Civelli and Deck (2018), we are able to create tasks of a desired cognitive difficulty by requiring someone to solve a series of puzzles. Acquiring a signal requires correctly solving two puzzles, with the exception of precision level 0 which requires solving one easy puzzle. ${ }^{13}$ Table 2 shows the precision level of the signal (first column), number of attributes changed in each puzzle that forms the task (second column) and the calibrated probability that a subject successfully acquires a signal (third column). In the experiment a subject could try as many times as she wished to acquire a signal. A subject could attempt to acquire a more precise signal after obtaining a less precise signal, but could not attempt to acquire a less precise signal after acquiring a more precise signal. ${ }^{14}$

\subsection{Experimental Implementation}

The experiment was written in Visual Basic, and it was deployed as a stand alone executable on computers at The Interactive Decision Experiment (TIDE) Laboratory at The University of Alabama. The experiment is a within-subject design, and it is broken into several parts. First the subjects read general instructions about how to interpret the prize table. Subject instructions are available in Appendix A. Subjects then went through 10 unpaid practice periods in which they selected one of 11 prize cards and observed the outcome from a randomly drawn prize number. After this, the subject read additional instructions that detailed signal acquisition and the logic puzzles. Subjects then completed a 10 minute unpaid practice phase in which they could solve puzzles to acquire signals, make prize card selections, and observe outcomes. After the unpaid practice phase, subjects reread the main instructions and then began the paid portion of the experiment. ${ }^{15}$

The paid portion of the experiment consisted of four phases. Each paid phase was associated with one of the four treatments described in the next subsection. The order of the treatments was randomized for each subject to control for learning and sequence effects. Subjects knew that there were four paid phases, but did not know anything about a given phase until reaching that point in the study. Each phase was preceded by brief phase

\footnotetext{
${ }^{13}$ Subjects do not have to solve the puzzle correctly to receive a precision level 0 signal since it is completely uninformative. Subjects do have to go through the motions of acquiring a level 0 signal to maintain consistency.

${ }^{14}$ This assumption encoded in the experimental setting captures the well-known principle in information theory that information cannot be forgotten. In order to avoid subjects having to rely on cognitive effort to remember the more informative signals, we prevent them from choosing signals with lower precision than the ones successfully obtained.

${ }^{15}$ The instructions before and after the practice phase differed slightly due to the subject being at different points in the study, but the two sets of instructions did not differ materially.
} 

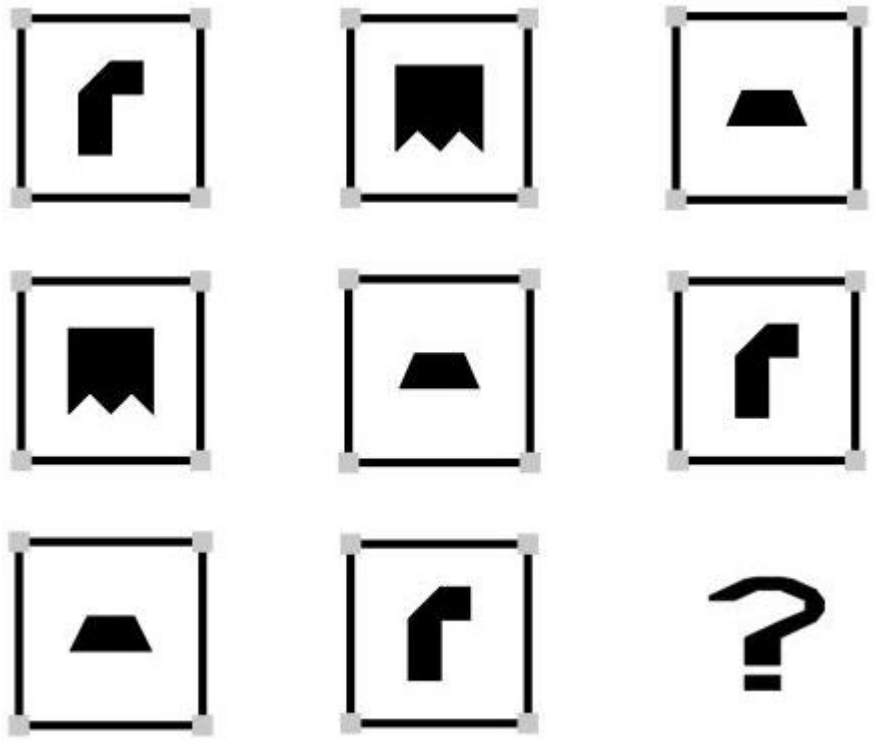

Figure 2: An example of a logic puzzle faced by the subjects. A 3-by-3 matrix of images with characteristics varying on multiple dimensions and with a missing element to be guessed to complete the puzzle.
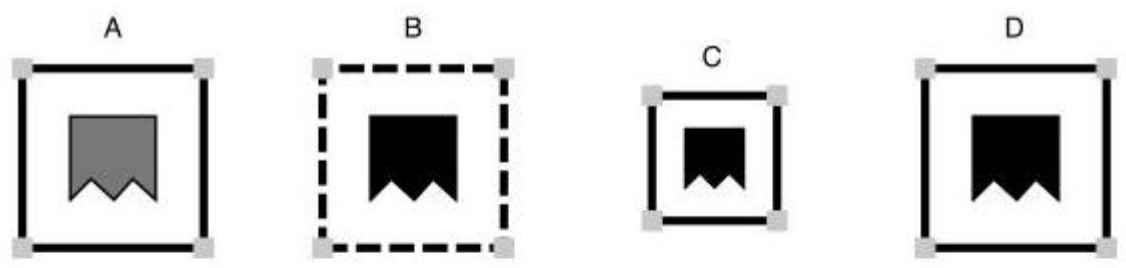

E

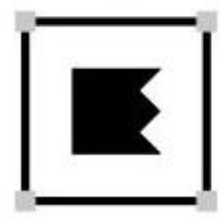

$\mathrm{F}$

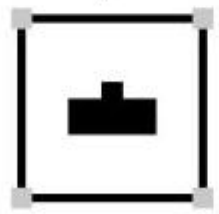

G

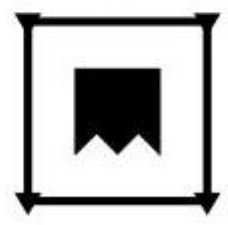

$\mathrm{H}$

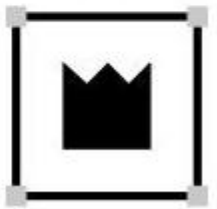

Figure 3: An example of solution set for the logic puzzle in Figure 2. The correct solution is option D. 
specific instructions. Each of the four practice phases lasted approximately 10 minutes. In each phase, subjects face an indefinite number of periods during which they can try to earn as much money as possible. The unspecified ending time is designed to mitigate end of game effects on behavior. A cap on the number of periods that could be completed during a phase was intended to discourage subjects from opting to always solve easy puzzles and race through the task. ${ }^{16}$ Once a subjects confirmed their prize card choice and received the payoff for that period, the subject proceed to the next period and hence next opportunity to earn money.

Subjects were recruited from a standing subject pool of undergraduate students at the University of Alabama. ${ }^{17}$ A total of 51 subjects participated in the experiment over the course of 3 sessions during October 2019. Average subject payment, including a $\$ 5$ participation payment, was $\$ 24.16$ USD. The salient portion of a subject's earnings was the sum of her cumulative earnings in the four paid phases. All prize payoffs displayed to the subjects were in cents and final payments were rounded up to the next quarter. Each session lasted approximately 90 minutes, which included working through the instructions, the practice phases, and participating in the actual experiment.

\subsection{Experimental Treatments}

Up to this point the description of the task has focused on the baseline (Treatment 1). In Treatment 2, which we dub the Delphic forward guidance treatment, the interest rate is drawn randomly from a uniform distribution whose support, [0.7 1.0], is lower than the support [0.4 1.3] of the baseline. The shrinkage of the support in a uniform distribution implies that the volatility associated to the interest rate is reduced. As a result, the degree of uncertainty about the economic environment and the level of risk in investing (savings) are both diminished.

In Treatment 3, dubbed the outlook deterioration treatment, we decrease the appeal of savings by decreasing the discount factor $\beta$ in (1) from $\beta=.9$ in Treatment 1 to $\beta=.75$. This corresponds to a situation where the agent becomes less optimistic about future investment

\footnotetext{
${ }^{16}$ For this reason, there was also a one-minute time penalty for subjects who made decisions without obtaining a signal precision above 0 . This feature of the experimental design exemplifies the case in which a representative agent incurs in a fee for investing her savings on a risky asset without any information about the return or loss of the investment. This cost is codified in the assumed functional form of the utility which, belonging to the quadratic family, delivers negative loss for risky investments. In the experiment, few subjects reached the period cap of 10 , but several opted to incur the time penalty from making a decision with a completely uninformed prior.

${ }^{17} \mathrm{~A}$ small percentage of the subject pool consists of graduate students from across campus.
} 


\begin{tabular}{|c|c|c|c|c|}
\hline & $\begin{array}{l}\text { (1) Range of } r \in \\
{\left[r_{1}, \ldots, r_{32}\right]}\end{array}$ & $\begin{array}{l}\text { (2) Discount } \\
\text { factor } \beta\end{array}$ & $\begin{array}{l}\text { (3) Utility function } \\
\text { parameters }[\bar{c} ; \alpha]\end{array}$ & $\begin{array}{l}\text { (4) Subjects' information } \\
\text { set before treatment }\end{array}$ \\
\hline $\begin{array}{l}\text { Treatment } 1(\mathrm{~T} 1) \\
\text { Baseline }\end{array}$ & {$\left[\begin{array}{lll}0.4 & 1.3\end{array}\right]$} & 0.90 & {$[1.5 ; 10]$} & General Instructions \\
\hline $\begin{array}{l}\text { Treatment } 2(\mathrm{~T} 2) \\
\text { Delphic Forward Guidance }\end{array}$ & {$\left[\begin{array}{lll}0.7 & 1.0\end{array}\right]$} & 0.90 & {$[1.5 ; 10]$} & General Instructions \\
\hline $\begin{array}{l}\text { Treatment } 3 \text { (T3) } \\
\text { Outlook Deterioration }\end{array}$ & {$\left[\begin{array}{ll}0.4 & 1.3\end{array}\right]$} & 0.75 & {$[1.5 ; 10]$} & General Instructions \\
\hline $\begin{array}{l}\text { Treatment } 4(\mathrm{~T} 4) \\
\text { Odyssean Forward Guidance }\end{array}$ & {$\left[\begin{array}{lll}0.4 & 1.3\end{array}\right]$} & 0.90 & {$[1.5 ; 10]$} & $\begin{array}{l}r \text { in top/bottom half of range, } \\
\text { correct with prob. } .9\end{array}$ \\
\hline
\end{tabular}

Table 3: Parametric characterization of each treatment. Column (3) specifies the parameter used in the utility function described in Section 2.1, where $\bar{c}$ is the satiation level and $\alpha$ the multiplicative constant. Column (4) states which information participants are given at the beginning of each treatment. Payoffs are displayed in Figure 1.

prospects. Finally, in Treatment 4, the Odyssean forward guidance treatment, we retain the same values of the lottery and range of interest rates as in the Treatment 1 but the realized draw becomes more predictable. Specifically, subjects are provided with a noisy clue as to whether the realized outcome is in the first half of the possible outcomes or the second half. This signal is correct with a $90 \%$ probability, which is known to the subject.

Table 3 summarizes the specific parametrizations for each treatment. The first column shows the range of the interest rate, while the second column the discount factor. In column 3 , we encode the parametrization of the utility function as described in Section 2.1, with $\bar{c}$ indicating the satiation level and $\alpha$ the multiplicative constant. Column 4 describes the clues the participants are given on the treatments they are about to play in the experiment, which characterizes their information set before they have made their signal and consumption choices.

\section{Results}

In this section we compare theoretical predictions to empirical observations. We start by illustrating the implications of the model assumptions laid out in Section 2 on the empirical setting and outcomes. We formally test how consumption choices of the representative rational inattentive (RI) agent compare with the choices selected by subjects and whether the behavior predicted by the RI model describes the empirical behavior better than the model with full information (FI). We proceed by formally testing the main theoretical 
predictions of the model in Section 2 against the empirical choices of participants in the laboratory experiment. First, we explore the value of information processing in observed consumption choices, and contrast empirical consumption outcomes with the theoretical optimum expressed in equation (7) of Section 2. This allows us to test the empirical distribution of consumption against the theoretical prediction in Subsection 2.2.1. Second, we test the effectiveness on both theoretical and empirical consumption outcomes of two kinds of forward guidance in the lab: Delphic and Odyssean forward guidance corresponding to the testable predictions in Subsection 2.2.2 and 2.2.4, respectively. Finally, we test whether consumption choices with a deterioration of the outlook as defined in Subsection 2.2.3 are aligned with their empircal counterparts.

\subsection{Theoretical Assumptions and Empirical Outcomes}

To establish the congruence between the model and the experimental set-up we compare average subject behavior and that of representative agent from the rational inattention model. Figure 4 shows how the average subject and the theoretical representative agent from the rational inattention model choose $c 1$ upon receiving a signal indicating that the interest rate is low (left-panel) or high (right-panel). Low interest rate refers to the first half of the possible realization of the interest rate, $[1,16]$, whereas high refers to the second half, $[17,32]$. Figure 4 shows a general consistency of empirical behavior with the model's predicted behavior across treatments. This result reassures us that the empirical framework under which the subjects operate is broadly consistent with the theoretical model. The difference between the two series likely stems from subjects' attitudes towards risk. While the model assumes that the representative agent is mildly risk averse (quadratic utility) and the payoffs presented in the experiment reflect this assumption, laboratory subjects may come to the experiment with different level of risk tolerance. As a result, the choices of the representative agent for $c 1$ potentially include signals with different precision level than the average subject.

The closed-form theoretical solution of the model in equation (7) relies on the assumption of quadratic utility of the agents. Translating this assumption into the empirical model results in a rather flat profile of expected gains, as evident from the payoff matrix in Figure 1. As stated in Section 2.2, while invoked mainly for analytical tractability, this assumption has both theoretical and empirical backing from the literature. ${ }^{18}$ Moreover, in the model with fixed lifetime endowment, consumption in period 1 is akin to investment in an asset with

\footnotetext{
${ }^{18}$ See, e.g., Bachmann et al. (2015).
} 

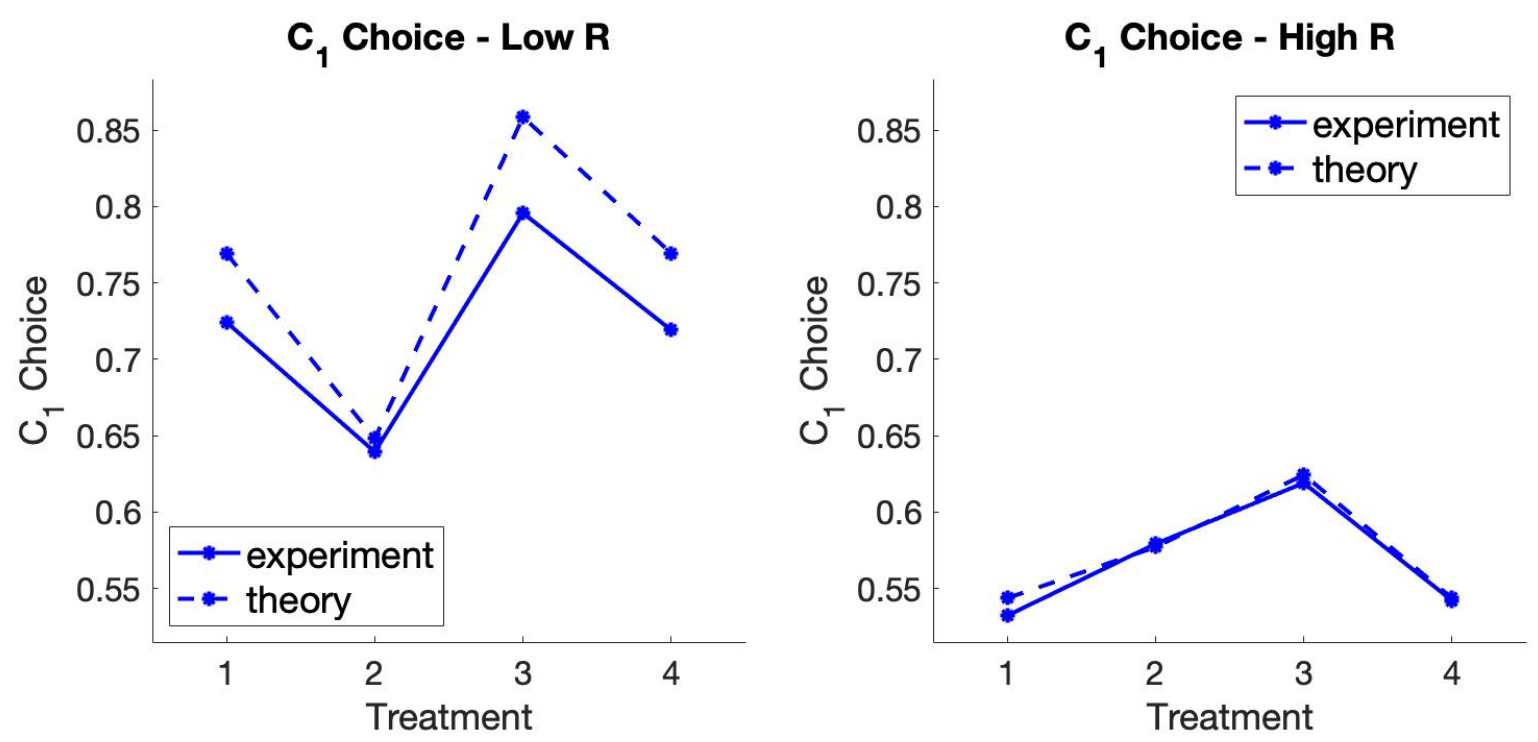

Figure 4: Consumption choices for the first period of the model $(c 1)$ conditional on observing a signal for low interest rate (left-pane) or high interest rate (right-panel), across treatments (x-axis). Solid lines correspond to experimental average across subjects, dashed lines are for the representative rationally inattentive agent.

certain return as opposite to the uncertain-return asset represented by savings - consumption in period 2 - implied by the stochasticity of the interest rate. In this environment, the theory predicts that when the utility gain of processing more information does not provide a sufficient compensation for the cognitive effort of precisely tracking the interest rate, the agent prefers to experiment with changes in the behavioral variable rather than varying signal acquisition.

Figures 5 and 6 illustrate that the result holds true in all the treatments. The figures plot individual (blue) and average (red) choices in the experiment across treatments for first period consumption, Figure 5, and precision, Figure 6. Consistent with the theory, THE laboratory evidence reveals that subjects prefer to exercise low cognitive effort in processing information. Instead, they are more prone to frequently change the behavioral variable in response to movements of interest rate.

In general, the model's predicted first period consumption behavior captures rather closely the average subject choices across treatments, as the black (representative agent) and red (experimental average) lines in Figure 5 show. This result has a stark monetary policy implication: in enviroments where deviations of interest rates policy are unimportant for people's utility, interest rate changes are unlikely to elicit behavioral reactions. 


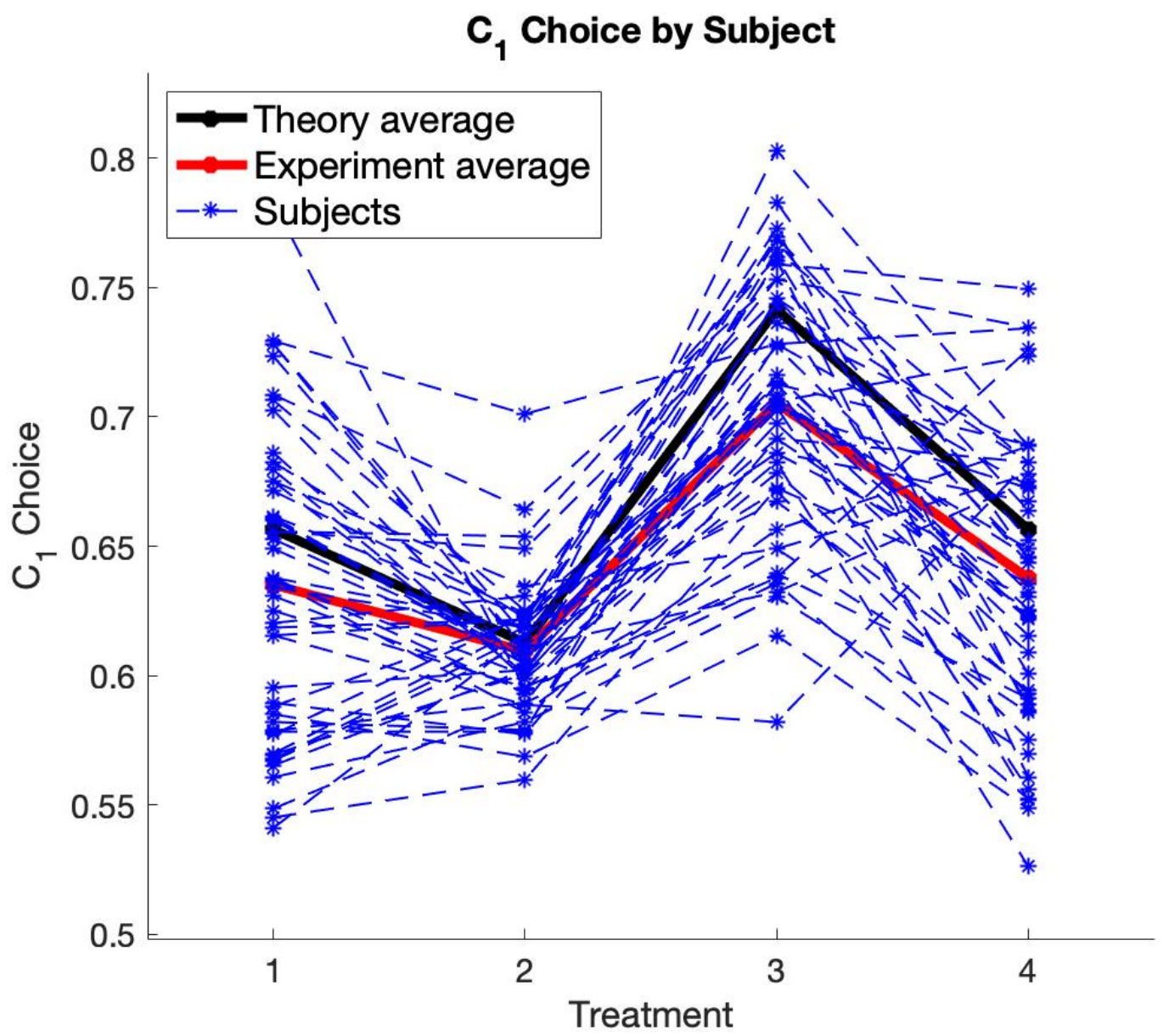

Figure 5: Consumption choices for the first period of the model (c1) across treatments for the subjects in the experiment. Individual average $c 1$ choices are given by the thin, dashed blue lines, overall subjects average by the thick red line, and the representative agent choice by the thick black line. 


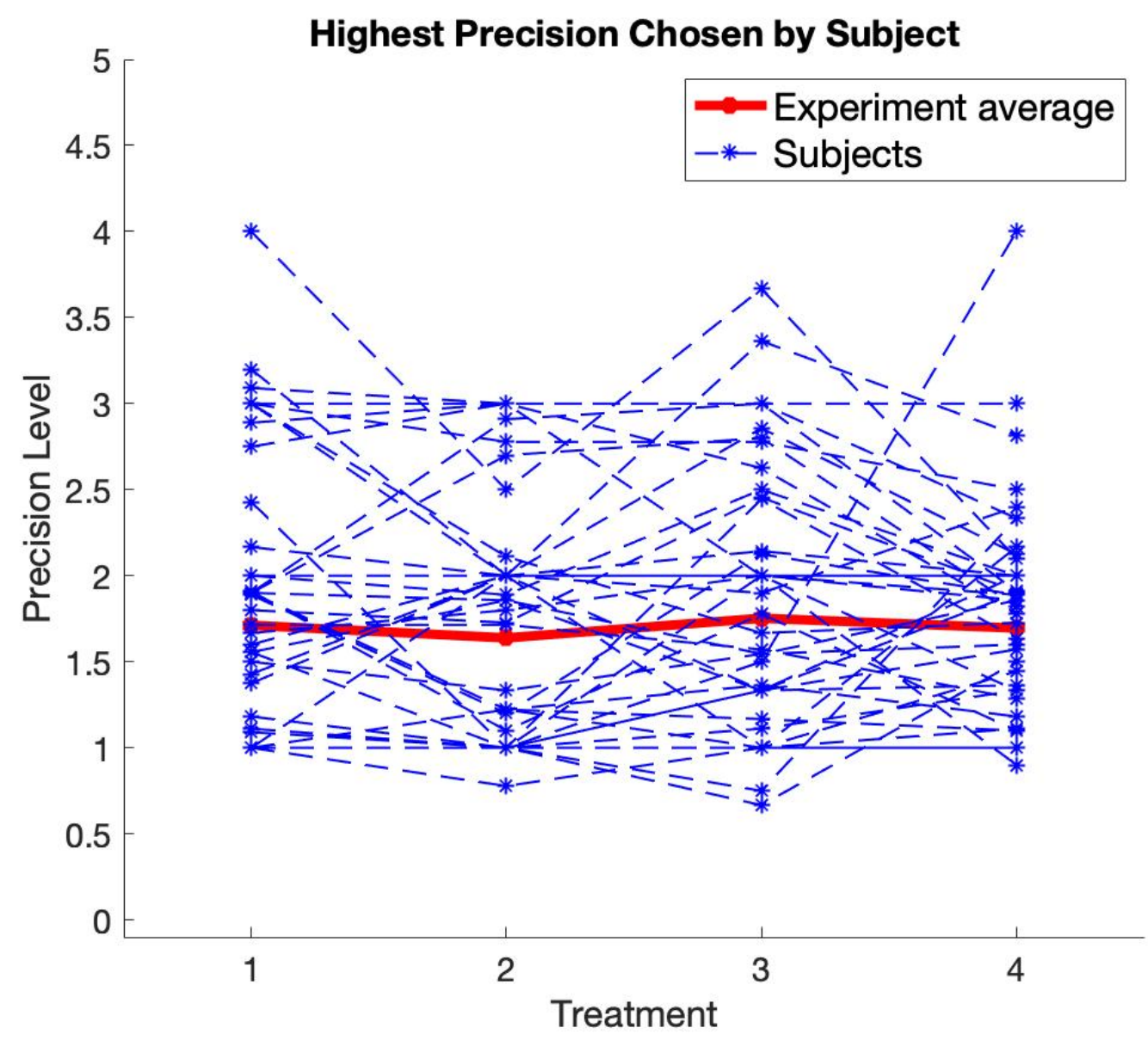

Figure 6: Highest signal precision level chosen by the subjects in the experiment before making their consumption choice across treatments. Individual average precision is given by the thin, dashed blue lines, while the overall subjects average by the thick red line. 


\subsection{Value of information, model validation and consumption outcomes}

We first establish how information about interest rate movements impact agents' total profits (lifetime utility) by comparing the laboratory outcomes with the full information benchmark for the baseline (i.e., Treatment 1: T1). Figure 7 shows the loss in lifetime utility calculated as the difference between realized total profits and full information profits as a percentage of full information profits (y-axis), plotted against the precision level of the signals acquired (x-axis). Each dot in the figure represents the average precision acquired by one subject and her average percentage profit loss in T1. From our experimental design, the participants can choose among six progressively higher precision levels, with 0 being the uninformative signal and 5 corresponding to the full information. It is immediate to see lower signal precision are associated with higher utility losses. We formalize this graphical intuition by estimating a kernel regression between the average loss and the average signal precision. The choice of a kernel regression is motivated by the possibility of a non-linear relationship between the two variables. ${ }^{19}$

Figure 7 also shows that there are diminishing return to information processing for the utility specification in equation (2.1) of Section 2.1. In fact, the flatness of the quadratic utility at high values of consumption provides minimal gains to information processing beyond signal precision 3, whereas for signals with precision less than or equal to 2 the lifetime utility gains from increasing information processing are more substantial.

To better understand the behavioral choices underlying these lifetime utility outcomes, Figure 8 compares the difference of consumption choices in period $1, c_{1}$, between the full information benchmark and the realized subjects' behavior. These experimental choices are plotted as a percentage of the full information benchmark against the average precision of the signal acquired. Figure 8 shows a lot of heterogeneity in the risk attitude of participants.

Inspecting the deviations in consumption reveals a pattern in participants' choices with respect to the full information benchmark that is a function of the precision selected. Subjects that acquire little information (below precision level 2) either under-consume in the first period with respect to the full information optimum or over-consume. Choosing a $c_{1}$ above full information reflects participants' under-confidence that the changes in interest rate will result into a gain that makes investment worthy. This precautionary consumption behavior arises because, with lack of precise information, risk-averse subjects prefer the safety of the certain outcome rather than the risky investment. Attention level and risk aversion are linked

\footnotetext{
${ }^{19}$ We use a local linear kernel with Nadaraya-Watson estimator and apply a bandwith of 0.58 , estimated following Bowman and Azzalini (1997).
} 


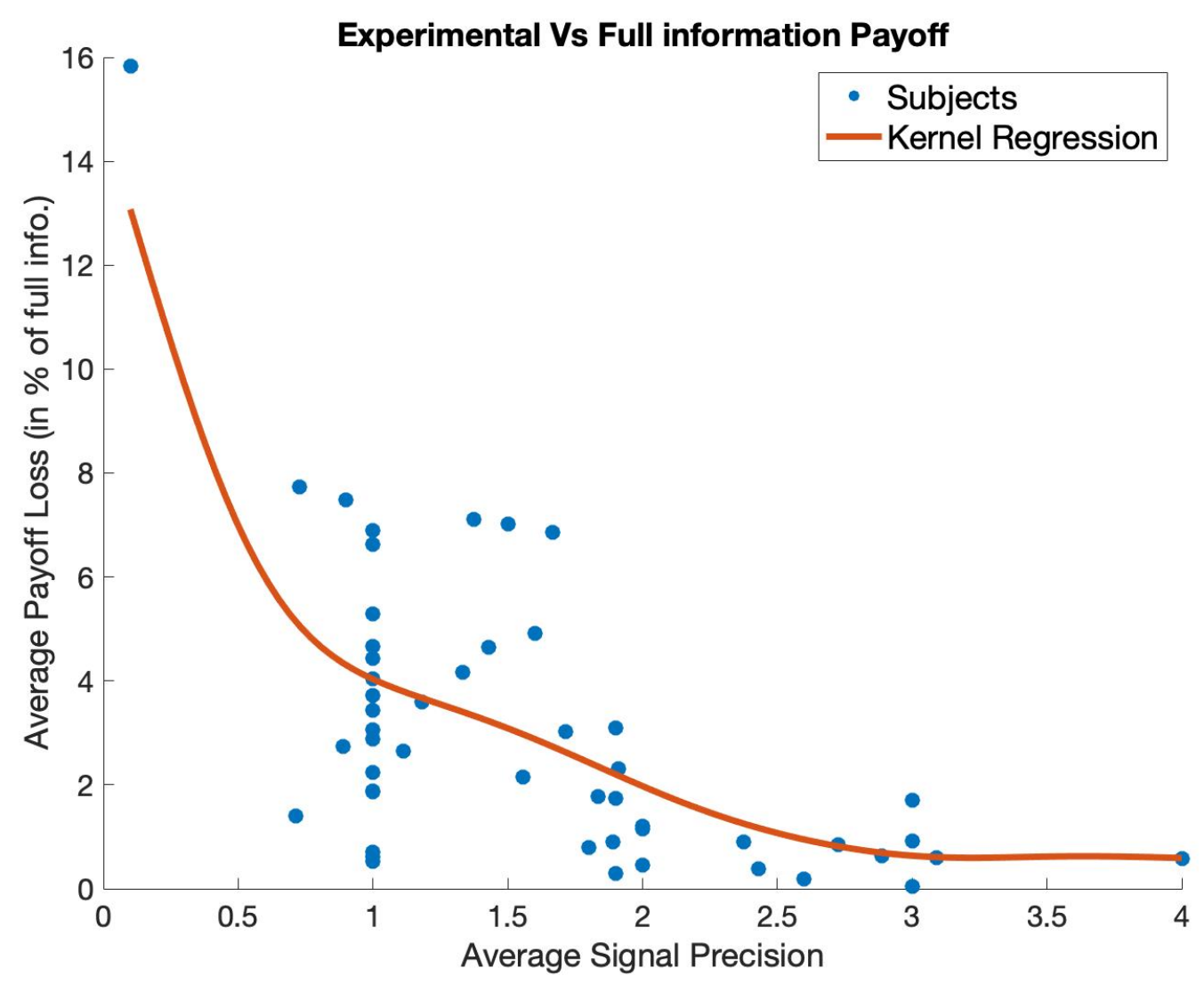

Figure 7: Average difference between full information and realized total payoffs (lifetime utility) as percentage of full information total payoffs plotted against average signal precision. The averages are taken within subjects. Each dot represents the average outcomes of one subject in the course of the baseline treatment. The red line corresponds to the local linear kernel regression with Nadaraya-Watson estimator.

as people with higher risk aversion tend to acquire higher precision signals. This point is a common theme in the rational inattention literature. ${ }^{20}$

Consistent with this argument, participants that acquire the highest signal precisions (level 2 and above) tend to be under-confident. By contrast, subjects under-consume when they feel buoyant about investment returns. These people tend to acquire low information and prefer to take chances on investment return. This behavior is consistent with higher risk tolerance.

Next, we evaluate the congruence of the subjects' behavior with that of the representative agent from the rational inattention (RI) model. Furthermore, we assess whether the consumption

${ }^{20}$ See, e.g., Tutino (2013). 


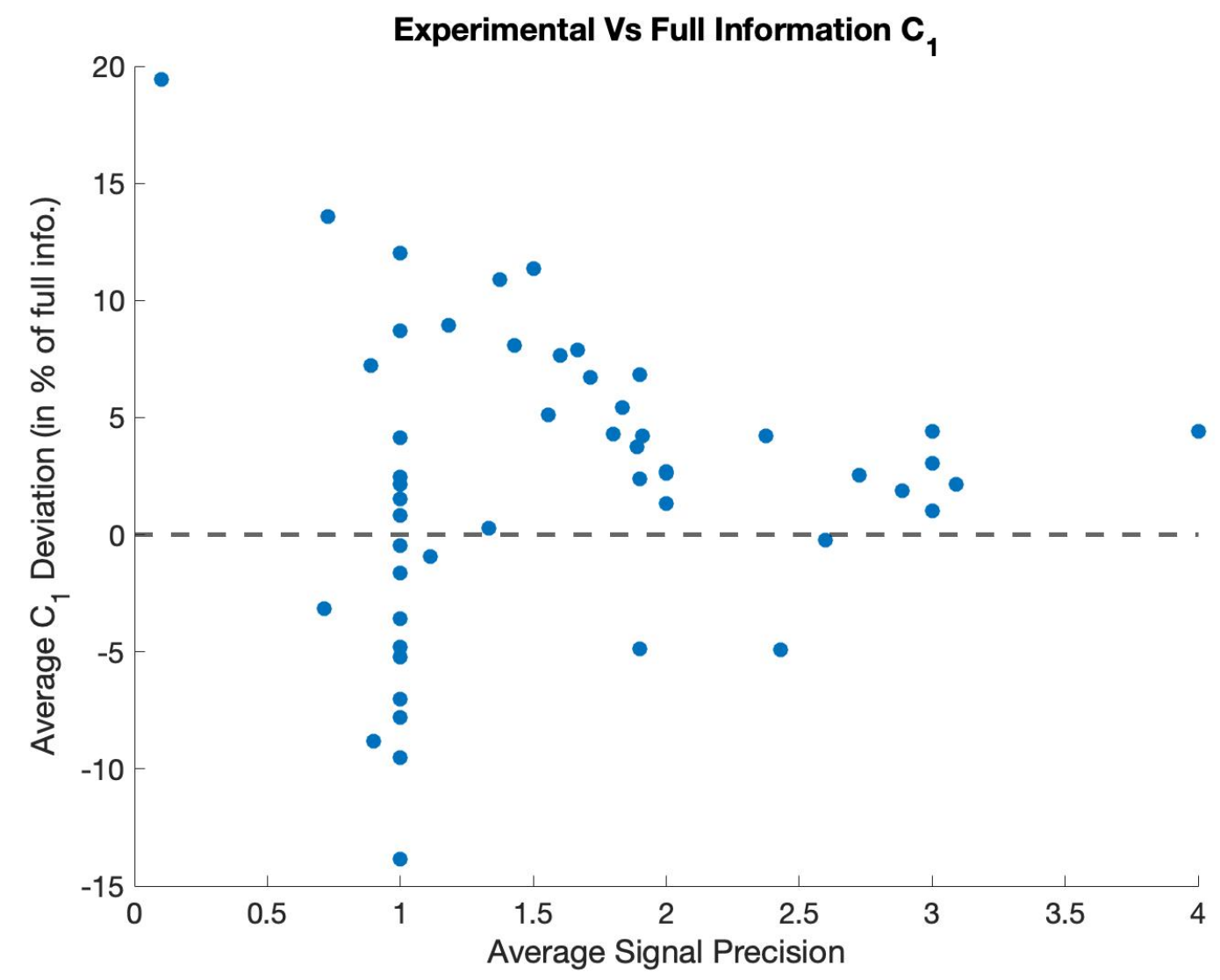

Figure 8: Average difference between full information and realized consumption in period $1, c_{1}$, as percentage of full information $c_{1}$, plotted against average signal precision. The averages are taken within subjects. Each dot represents the average choices of one subject in the course of the baseline treatment.

behavior predicted in the RI model describes the empirical consumption choices better than the full information (FI) model.

Figure 9 shows a scatter plot of the consumption choices $c_{1}$ of the RI representative agent (y-axis) against the empirical choices of the subjects (x-axis). For both theory and experimental data the $c_{1}$ outcomes are demeaned and each dot represents an observation of the triplet [RI $c_{1}$, Empirical $c_{1}$, Signal Precision]. ${ }^{21}$ The color of the dot indicates the precision chosen by each subject according to the scale on the upper right corner of the figure. The size of the dot is proportional to the sample frequency of each triplet. There are two main take-aways from this figure.

First, as we have seen in Figure 8, for higher precision levels (i.e., level 4) the empirical

\footnotetext{
${ }^{21}$ For the baseline model, $\mathrm{T} 1$, the mean for the RI $c_{1}$ is 0.65 whereas the empirical average is 0.63
} 


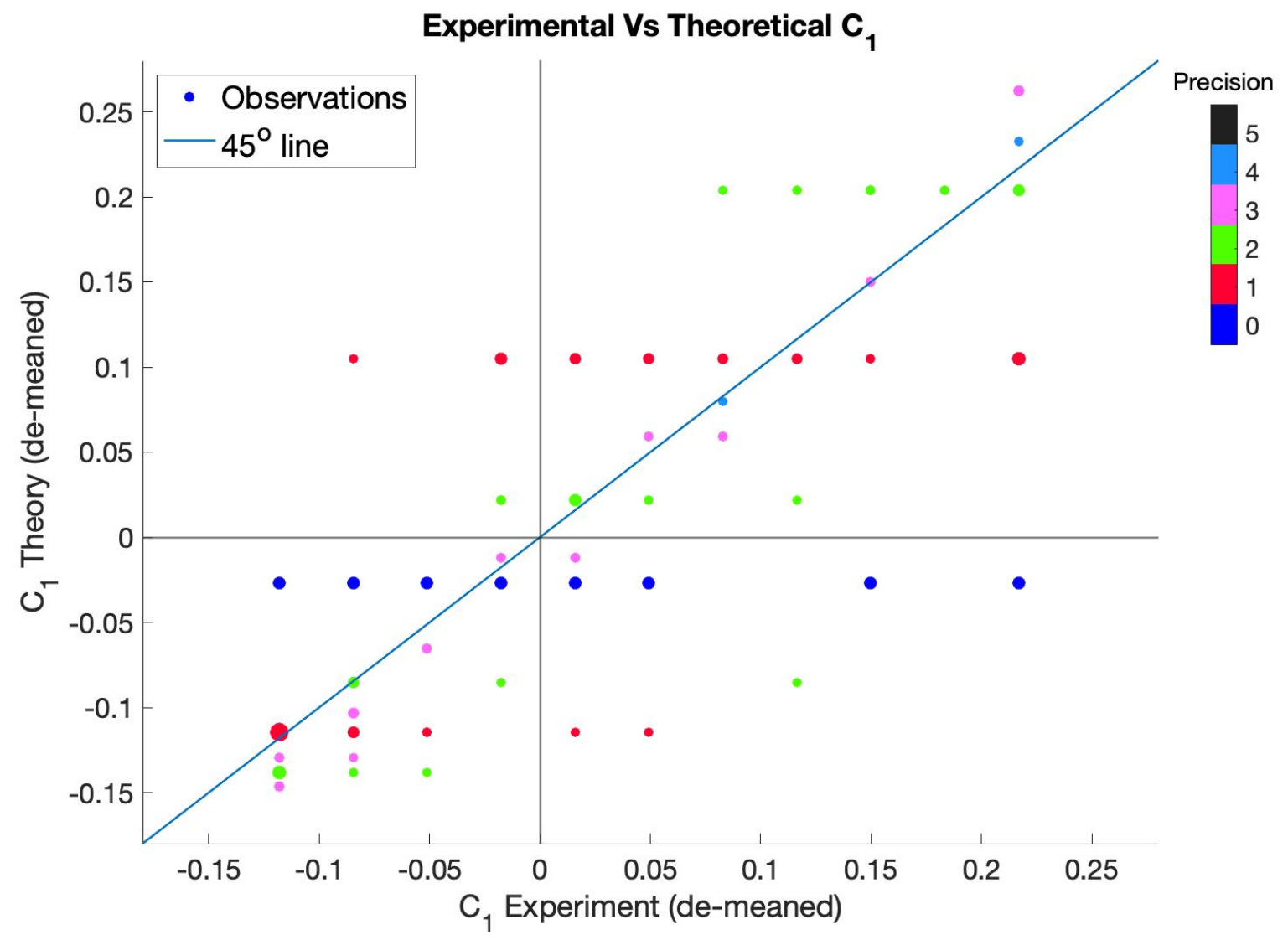

Figure 9: Scatter plot of theoretical and experimental consumption choices, $c_{1}$. Observations are de-meaned and each dot represents a subject. The color of the dots indicates the precision selected by the subject according to the legend in the upper-east corner of the figure. The size of the dot is proportional to the sample frequency of a given triplet (precision, theoretical $c_{1}$, empirical $\left.c_{1}\right)$.

choices $c_{1}$ are very close to both the FI model and the RI representative agent choices. This observational equivalence between the FI model, the RI model, and observed behavior arises in our setting because of the length of the interval revealed by the signal for high precision compounded with the flatness of the utility function assumed in the theoretical model. Thus, the more informative cases in terms of congruence of model predictions and empirical choices occur at lower precision levels where the width of the interval is substantial and the sample much more sizeable.

Second, with respect to informative precision levels ranging from 1 to 3 , Figure 9 shows a remarkable consistency of the empirical outcomes to the behavior predicted by the RI model for the cases with higher sample frequency (i.e., bigger dots). This finding places the average 
consumption choice per subject strikingly close to the RI representative agent outcome in each precision level.

We corroborate this visual insight by formally testing the fit of the RI model in describing the experimental data and its performance in comparison to the FI outcomes. To this end, we run linear regressions of individual experimental subjects' $c_{1}$ choices on the RI representative agent behavior predicted by the model. We first run these regressions individually for the three informative precision levels, precision 1 to 3 , observed in the baseline treatment of the experiment, $\mathrm{T} 1 .^{22}$ We then estimate two pooled models, again for the T1 observations: one including fixed effects and one without fixed effects but using weights proportional to the length of the signal intervals. ${ }^{23,24}$ We run this battery of regressions of the empirical $c_{1}$ 's on the full information solution as well, and we formally contrast the regressions outcomes from the RI and the FI models to assess their relative performance in explaining the experimental data. The results of this analysis are in Table 4.

The first three models of Table 4 present the estimates of an OLS regression of the empirical consumption, $c_{1}$, on either the rational inattention prediction of consumption, $c_{R I}$ in the table, or the full information outcome of consumption, $c_{F I}$, conditional on the precision levels of the signal. In all the regressions we use robust standard errors clustered by subjects. We take the evidence from these three models as the baseline in our analysis. If a theoretical model is a good fit for the data, we would expect the slope associated to the consumption choice predicted by the model to be close to one and the constant of the regression to be close to zero. As the table illustrates, the coefficients associated to $c_{R I}$ are systematically bigger and closer to 1 than those associated to $c_{F I}$. A t-test of the slope of the regression cannot reject the hypothesis that the slope of the regression for the model is, in fact, one for precision 1 but not for the other two. Similarly the constant terms are also smaller in the RI case, and not significantlly different from zero for precision 1.

These results are confirmed by the other two models in Table 4. Model (4) presents the results of the linear regression for unconditional $c_{1}$, estimated with precision fixed effects

\footnotetext{
${ }^{22}$ We omit precision level 0 , the uninformative signal, since the theoretical prediction for this particular signal corresponding to a zero-capacity Shannon's channel is just a random draw from the uniform distribution of the interest rate.

${ }^{23}$ The F.E. model excludes precision 4, since only one subject chooses this signal in the experiment and only 4 observations are available at this precision.

${ }^{24}$ The F.E. model controls for the systematic bias in the distribution of consumption at different precision levels. For instance, both RI models and the data display leptokurtic and left-skewed distributions as opposite to the uniform updated prior revealed by the signals. The weighted model is meant to capture the fact that subjects face more freedom in the choice of $c_{1}$ for lower precision signals. The probability of a choice being close to the RI model simply because of chance is therefore smaller the larger the interval.
} 
to account for systematic biases within precision. Model (5) presents the results for the unconditional $c_{1}$, where the observations are weighted in proportion to the length of the precision interval. This weighting scheme accounts for the fact that a larger interval revealed by the signal is relatively more informative of the goodness of fit of the $R I$ model than a smaller one. The slope of the regression is not significantly different than one only for the RI case in Model (5), however the constant is not significantly different than zero for both the RI regressions, but substantially larger and significant for the two FI models. Interestingly, the fixed-effect models show there is little, if no, heterogeneity in the pooled regression across precision levels.

The last rows of the table formally test whether the rational inattention model does a better job in predicting the empirical data then the full information model, thereby testing the assumption of capacity constrained individuals. The BIC statistics show that in the pairwaise comparison within each regression group, the RI model exhibits a better fit than the FI model and it would always be strongly selected by the criterion. ${ }^{25}$ The DavidsonMcKinnon (J-test) and Cox-Pesaran tests for non-nested model comparison favor the rational inattention model over the full information model across the board as well. At the same time, the alternative hypothesis that the the FI model is preferable is strongly rejected in each case (not reported in the Table). Thus, we can reject the hypothesis that individuals behave according to the full information model and we find robust evidence that our subjects display a degree of rational inattention in their consumption choices.

${ }^{25}$ The AIC results tell same story, thus we omit them from Table 4 for sake of economizing on space. 
Table 4: Model validation - Estimation of the regression of Empirical consuption choices, $c_{1}$, on the consumption predicted by the Rational Inattention model, $c_{R I}$, and by the Full Information model, $c_{F I}$. OLS estimation, with standard errors clustered by subject reported in parenthesis. Significance of estimated coefficients at 1\%, $5 \%$, and $10 \%$ level is respectively indicated by ${ }^{*},{ }^{* *}$, and ${ }^{* * *}$. Models (1) -(3) are estimated by precision level of the selected signal as indicated in the Table. Model (4) uses precision fixed effects, with precision 0 as baseline and excluding precision 4. Model (5) is a weighted pooled model, with weights proportional to the length of the signal intervals. ${ }^{(a)}$ T-Test of the null hypothesis that coefficient on either $c_{R I}$ or $c_{F I}$ is equal to one (p-values reported); ${ }^{(b)}$ BIC statistic computed using N. Obs.; ${ }^{(c)}$ Nonnested model coparison tests. J-Test correspond to the Davidson-McKinnon test (p-values reported). The alternative hypothesis $M_{F I} \succ M_{R I}$ always rejected (omitted).

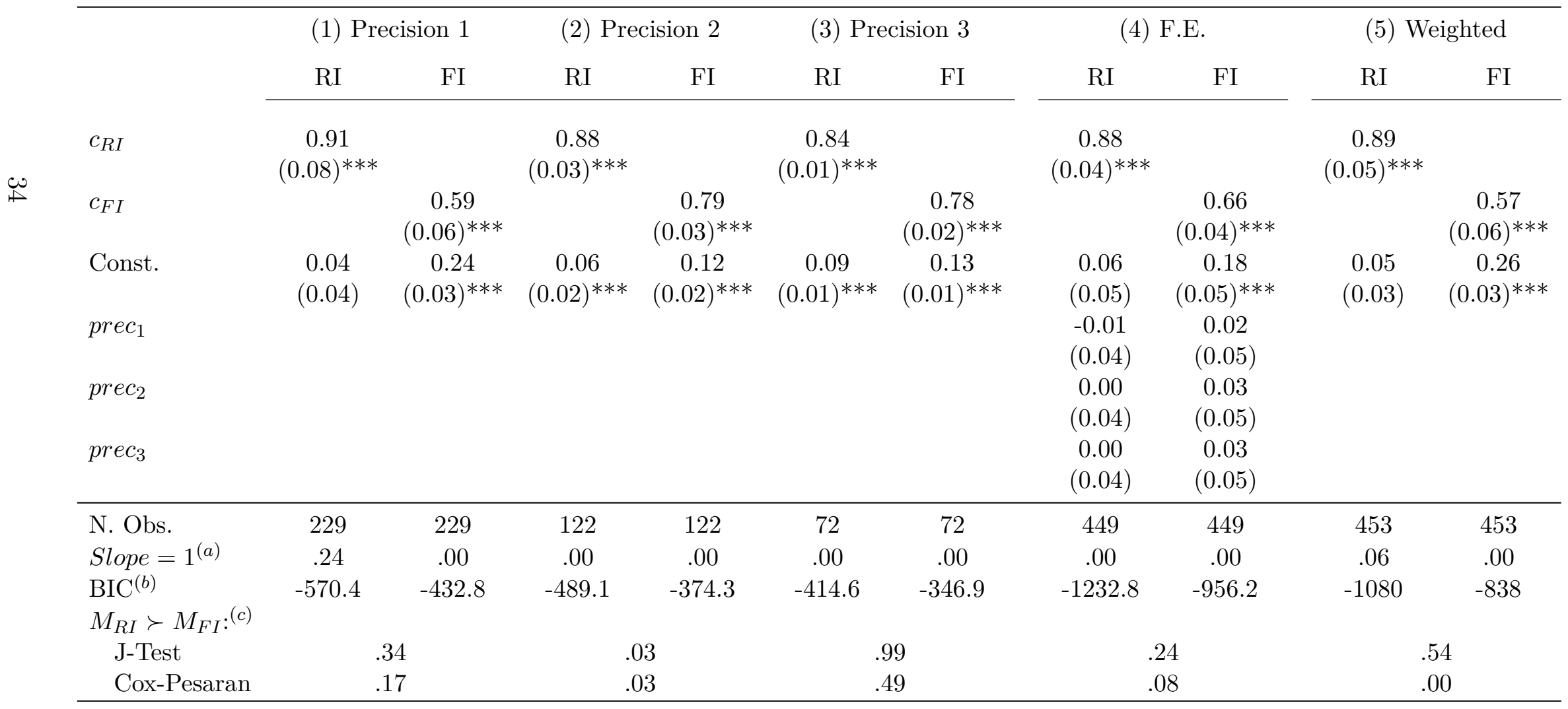




\subsection{Forward guidance and consumption outcomes}

We explore the effects on consumption and information processing of two dimensions of forward guidance. First, we study whether a monetary authority that commits to lower volatility of the policy instruments succeeds in affecting consumption behavior of the agents as well as modifying their attention to monetary policy. This treatment can be considered as a form of Delphic Forward Guidance. ${ }^{26}$ We test the theoretical prediction in Subsection 2.2.2 in the laboratory experiment by giving to the subject a significantly lower range of possible realizations of the interest rate, thereby reducing the variance of the (uniform) interest rate distribution. The results of this stimulus are in Subsection 4.3.1.

Second, we study whether an enhanced predictability of the interest rate policy may provide enough stimulus to the subject to change their behavior toward consumption and information processing. This treatment can be considered as a form of Odyssean Forward Guidance. ${ }^{27}$ We test this assumption by having the monetary authority announcing whether the monetary policy stance would be accomodative (low interest rate) or tight (high interest rate) during the current period and committing to the announcemnet $90 \%$ of the time while deviating from that commitment with probability 10\%. The results of this stimulus are in Subsection 4.3.2.

\subsubsection{Delphic forward guidance and consumption outcomes}

A monetary policy that makes the interest rate path less volatile and, as a result, more stable by, e.g., providing forward guidance via Fed officials' communication and regular release of the Summary of Economic Projection (SEP) forecasts, requires less effort from the representative agents to track the policy instrument closely. This occurs because the predictability of the interest rate path implies a lower cognitive burden to the tracking of the interest rates for the agents. As a result, agents are more confident in their estimate of the monetary policy stance when making consumption and investment decisions. In this scenario, the model predicts an increase in first period consumption when interest rate is low and a decrease of first period consumption when interest rate is high as stated in Subsections

\footnotetext{
${ }^{26}$ Campbell et al. (2012) defines Delphic forward guidance as using interest rate to stabilize the economic outlook, while Odyssean forward guidance is a commitment on undertaking policy actions in the future, e.g., "keeping rates lower for longer" when inflation and GDP rise.

${ }^{27}$ We see this treatment as a form of Odyssean forward guidance in that, at least in principle, providing a signal on the monetary policy stance and committing to a high accuracy of the signal is a guidance to the private sector on the behavior of the interest rate that may foster a more predictable environment in which to consume and allocate attention.
} 


\subsection{1 and 2.2.2.}

We implement this scenario with a reduction of the interest rate volatility to about one tenth of the baseline one, as explained in Section 3.4. This treatment captures the monetary policy's aim to stimulate private sector's investment by providing a stable economic environment for households and lower their cognitive effort of tracking the interest rate. ${ }^{28}$

We evaluate the effectiveness of this policy experiment by comparing deviations of lifetime utility with respect to the full information benchmark as a percentage of full information values in this scenario with respect to the baseline. As standard for evaluating monetary policy changes in the literature, we use lifetime utility as proxy for agents' welfare, measured by the mean total profits of each subject in the experiment. Figure 10 illustrates the kernel estimates of the aggregate distributions of the welfare losses for the rational inattention theory (top panel) and the experimental data (bottom panel). In both panels, blue dashed lines indicate the baseline (T1) whereas the red solid lines represent the Delphic forward guidance treatment (T2). The densities are estimated using a Gaussian kernel.

Four main observations emerge from this figure. First, the experimental data is strongly consistent with the RI theoretical predictions. The main difference between experimental and theoretical cases is a longer tale for the experimental distributions. Second, welfare losses are largely smaller under T2 than they are under T1. The T2 density is much more concentrated than the T1 one: the empirical density displays a clear, sizeable peak around $0.15 \%$, while the distribution of losses in $\mathrm{T} 1$ is spread out over the support $0-10 \%$ with a peak around $1.25 \%$. More importantly, the standard deviation of the distribution of losses is dramatically reduced from $2.6 \%$ in $\mathrm{T} 1$ to $0.7 \%$ in $\mathrm{T} 2$. The $\mathrm{T} 2$ distribution is leptokurtic and left-skewed.

Third, the difference between RI theoretical outcomes and experimental data can likely be attributed to risk aversion. The representative RI agent displays modest risk tolerance consistent with the quadratic utility, whereas experimental subjects appear more risk averse. However, note that the average subject in the experiment modestly lowers her attention to the interest rate (decrease signal precision, as evident from Figure 6) and moderately increase investment (lowers $c_{1}$ consumption with respect to T1 as shown by Figure 5). With respect to $c_{1}$ and investment decisions, the representative RI agent and the average experimental subjects appear alligned as per Figure 5.

Finally, in the RI theory as well as in the experimental data, the decrease in both the

\footnotetext{
${ }^{28}$ Under our distributional assumption on the interest rate, a reduction in its variance implies that fewer bits of information are needed to accurately track the interest rate with respect to the baseline.
} 


\section{Rational Inattention Theory}

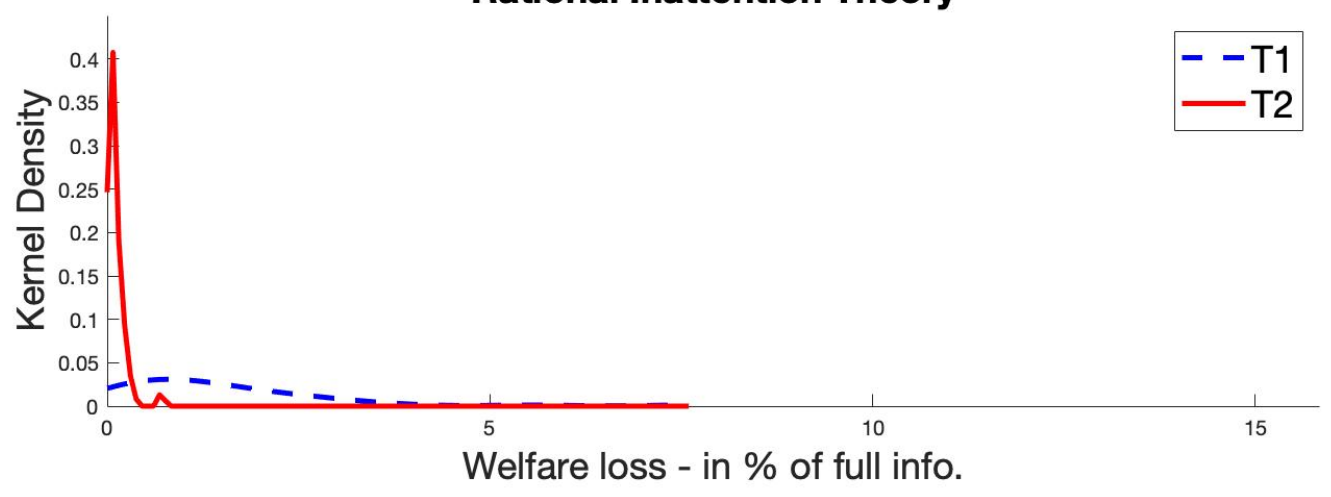

Experimental Data

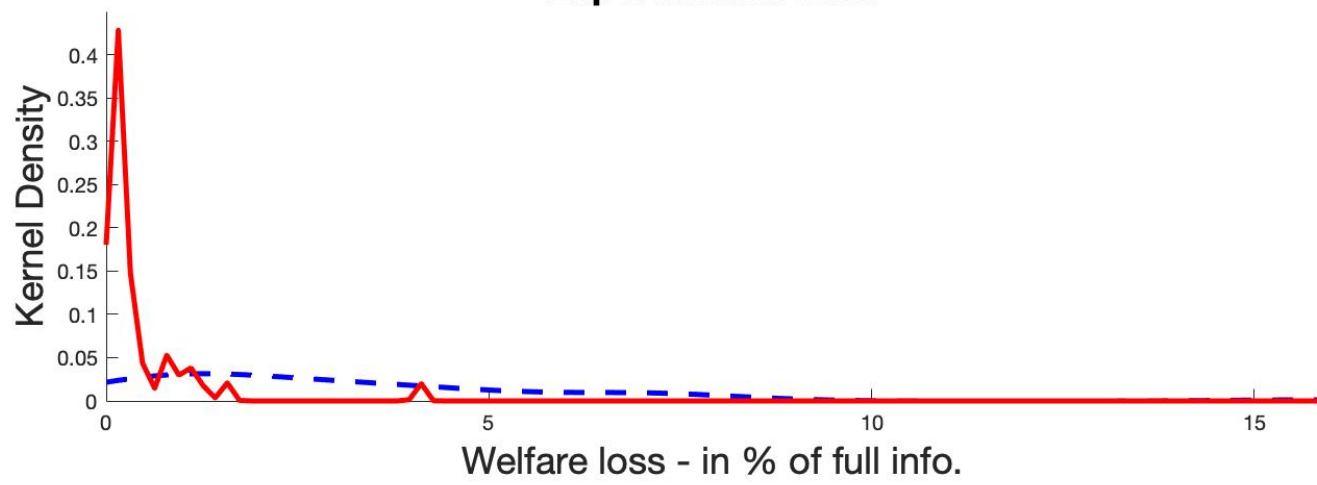

Figure 10: Aggregate density distribution of welfare losses as percentage of full information under the Delphic forward guidance treatment (T2) in red solid line and the baseline (T1) in blue dashed line. The aggregate densities are estimated using a Gaussian kernel. The top panel shows the density for the representative RI agent, whereas the bottom panel displays the density for the average aggregate experimental data. For the data, welfare losses are computed as mean deviations per subject of total profits from full information profits.

magnitude and the volatility of welfare losses are consistent with behavioral choices that are more deliberate and attune to an economic environment with reduced uncertainty with respect to the baseline. The monetary policy implication of the comparison between T2 and the baseline is that the form of Delphic forward guidance considered appears to be effective in reducing the welfare loss in a world where agents are rationally inattentive.

\subsubsection{Odyssean forward guidance and consumption outcomes}

An announcement of future paths of interest rate constitues an attempt from the monetary authority to keep monetary policy predictable and, as a result, ehancing the predictability 


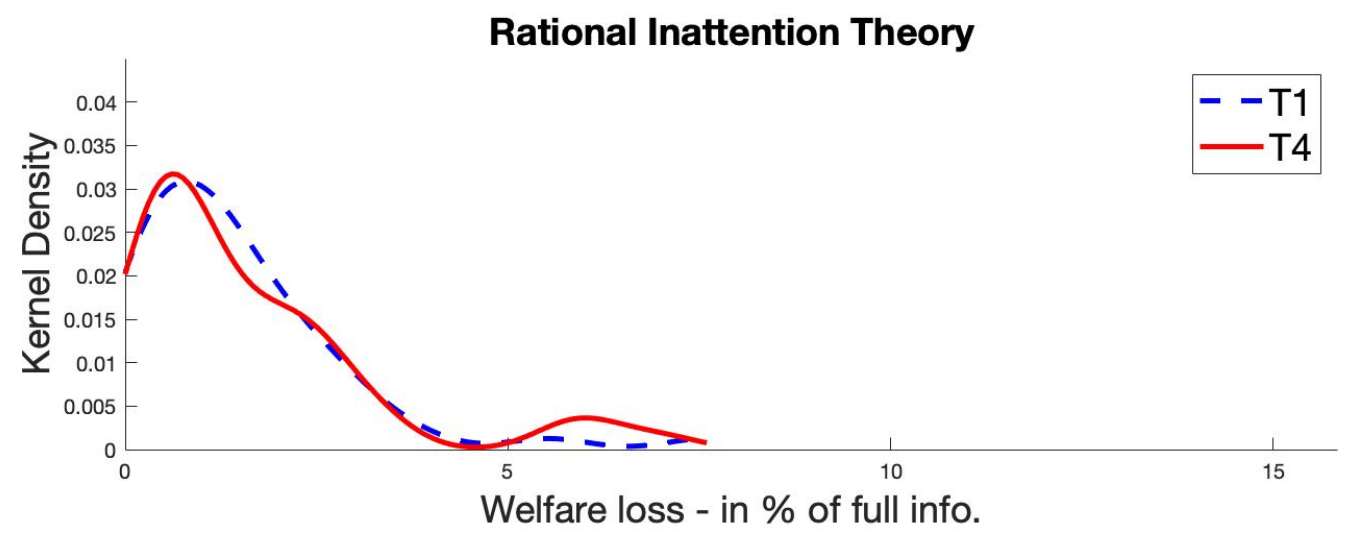

Experimental Data

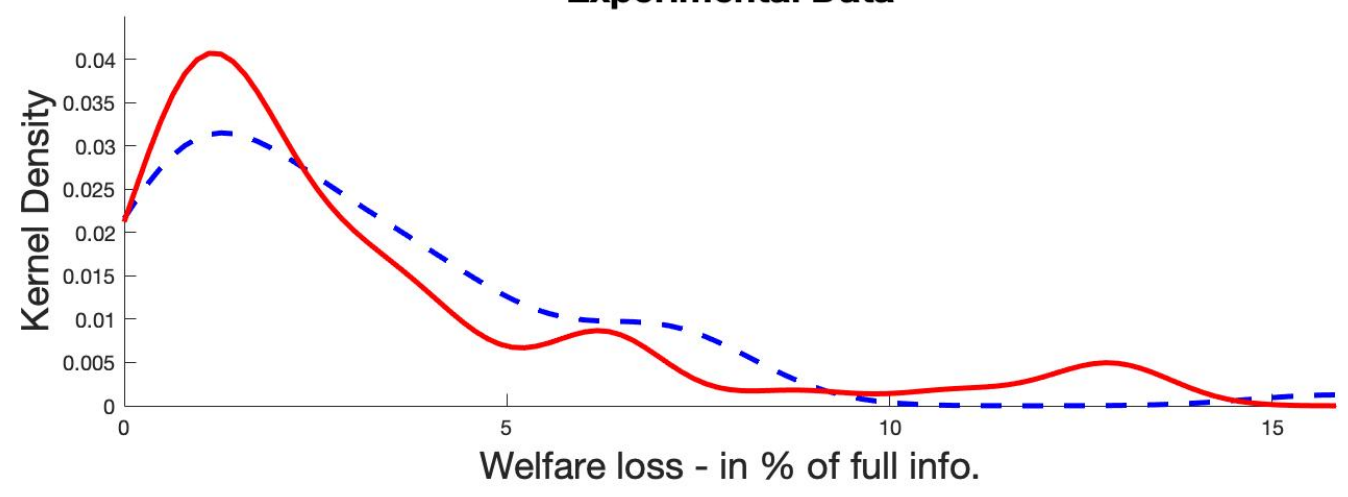

Figure 11: Aggregate density distribution of welfare losses as percentage of full information under the Odyssean forward guidance treatment (T4) in red-dashed line and the baseline (T1) in blue solid line. The aggregate densities are estimated using a Gaussian kernel. The top panel shows the density for the representative RI agent, whereas the bottom panel displays the density for the average aggregate experimental data. For the data, welfare losses are computed as average mean deviations per subject of total profits from full information profits. 
of the economic environment. Examples of this form of forward guidance in the U.S. are in several FOMC's statements and speeches signalling whether the monetary policy stance is loose or tight. The literature has labelled this kind of forward guidance Odyssean forward guidance.

For this scenario, we assume that the central bank announces to the public whether the monetary policy stance in the current period would be accomodative (low interest rate) or tight (high interest rate). The monetary authority commits to deliver a signal on the monetary policy stance that is $90 \%$ accurate. For this case, the model predicts no change in consumption behavior with respect to the baseline. The rationale behind this theoretical finding stems from the fact that, conditional on the signal acquired, the optimal choice in equation (7) remains unchanged under this scenario with respect to the baseline.

We implement this scenario by giving the subjects the announcement on the monetary policy stance at the beginning of each period of this treatment, as explained in Section 3.4. This treatment is meant to replicate the commitment of the monetary authority to make the interest rate more predictable. This policy's goal is that interest rate rigidity may foster investment while lowering the cognitive burden of keeping track of the policy rate. As we did for the previous form of forward guidance, we assess the effectiveness of this policy experiment on private sector's welfare by studying the percentage deviations of lifetime utility from the full information benchmark in this treatment in comparison to the baseline.

The estimated aggregate kernel densities of these deviations are reported in Figure 11 for the rational inattention theory (top panel) and the experimental data (bottom panel). As above, blue dashed lines indicate the baseline (T1), whereas the red solid lines represent the Odyssean forward guidance treatment (T4). We use the same estimation methodology to derive this figure as we did for Figure 10.

From Figure 11 it is clear that the theoretical and experimental distributions are once again strongly consistent with each other. It is also immediate to note that the distributions of welfare losses are remarkably similar under T1 and T4. We formally test for the equivalence between these two distributions by using the two-sample Kolmogorov-Smirnov (KS) test. The null hypothesis tested is that the two vectors of welfare losses from the two treatments are from the same continuous distribution, evaluated against the alternative hypothesis that the data are from different distributions. ${ }^{29}$ The null hypothesis is not rejected at very

\footnotetext{
${ }^{29}$ The Kolmogorov-Smirnov test is a nonparametric method which compares the cumulative distribution functions of two data vectors and is based on the maximum absolute distance between the empirical distributions of the two samples. It is sensitive to both location and shape of the distributions.
} 


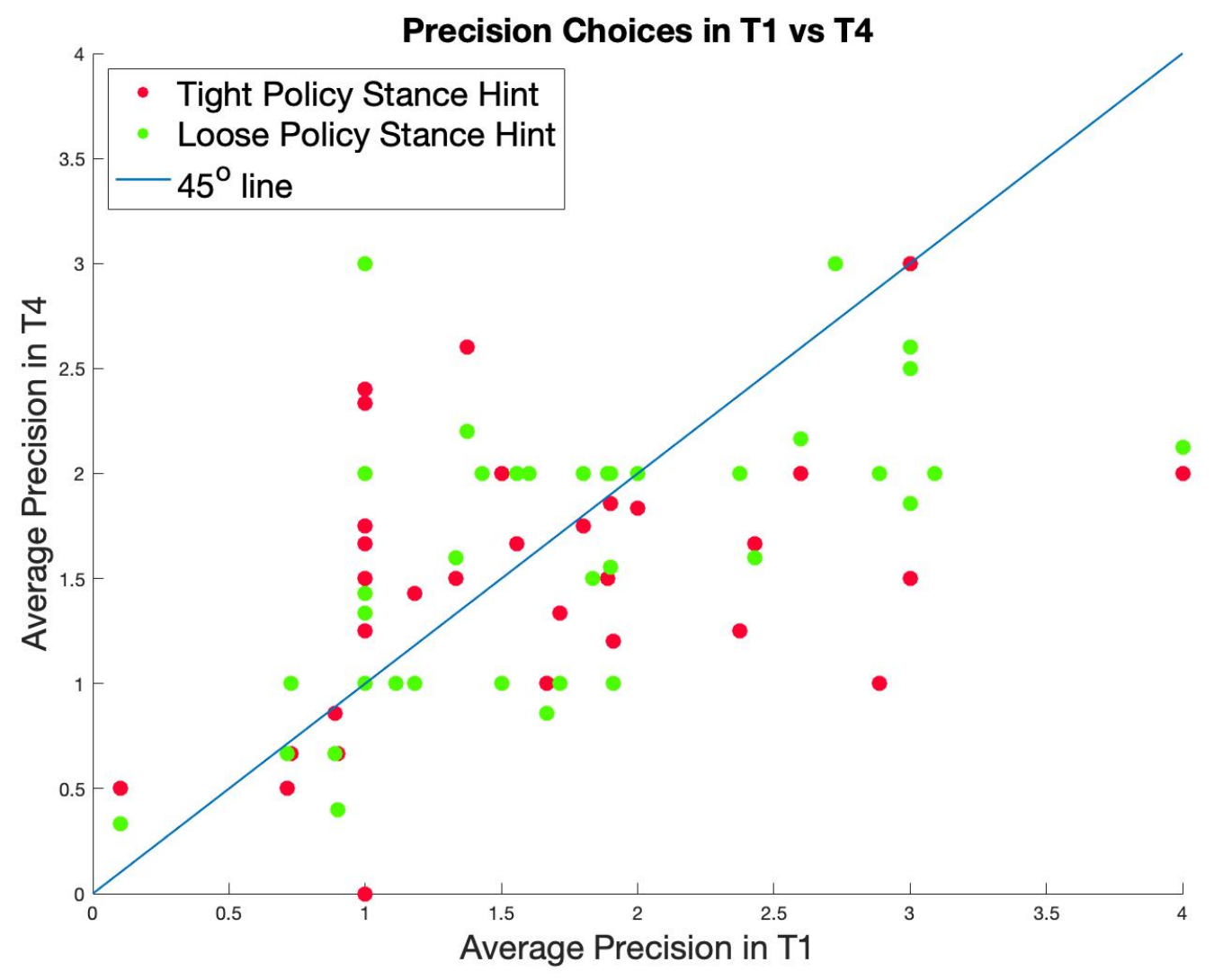

Figure 12: Average precision by subject in T1 vs. T4. Each dot represents the average precision chosen by an individual. For each subject, the average is calculated across realizations of the signal that $r$ is high (top half of the support of $r$ ) in red, or low (bottom half of the support of $r$ ) in green.

high level of confidence (p-value of .95), while the same test conducted to compare the distributions of T1 and T2 strongly rejects the null (p-value of .00).

To shed further light on how this treatment affect subjects' choices and behavior with respect to the theoretical predictions for the representative RI agent discussed in Subsections 2.2.4, we look at participants' decisions of precision next. Figure 12 shows the average precision chosen by a subject in T1 vs. T4. For each subject, two averages are calculated. One corresponds to the decisions taken after receiving a central bank clue for tight monetary policy stance (high $r$, i.e., $r$ in the top half of the support), and is represented by red dots. The second is for the decision taken after receiving a clue for loose monetary policy (low $r$, i.e., $r$ in the bottom half of the support), and is denoted green dots.

Figure 12 illustrates two main observations. First, participants do not systematically 
modify their information acquisition according to whether the monetary poilicy stance is tight or loose, as the lack of a clear pattern of changes in information gathering from T1 to $\mathrm{T} 4$ in response to high and low interest rates shows.

Second, the figure also suggests that the decision to change information gathering might be triggered by their cognitive ability. Subjects comfortable processing very little information in $\mathrm{T} 1$ (precision $\leq 1$ ) treat the signal as a new prior where the tracking of the interest rate is made easier by the shrinkage of the support from 32 values to 16 values with $90 \%$ probability. ${ }^{30}$ For these low-cognitive capacity subjects, the Odyssean forward guidance treatment elicits a modest increase in attention and cognitive effort in tracking the monetary policy instrument from $\mathrm{T} 1$ to $\mathrm{T} 4$.

On the contrary, for people who select relatively more informative signals in T1 (precision $>1$ ), the central bank's signal on the policy stance provides a mild incentive to lower their attention to the interest rate. While their chosen signal is still more informative than the one provided by the monetary authority, they marginally drop the precision of their optimal signal following the announcement. Since our model's testable predictions are focused mostly on behavioral outcomes induced by information gathering, rather than the information gathered before the decisions are made per se, we leave a more detailed investigation of these patterns for future research. ${ }^{31}$

The litmus test of whether this form of Odyssean forward guidance is successful in modifying the economic behavior of the subjects, however, ultimately rests on the ability of the policy to affect macro aggregates, such as consumption and investment and, as a result, welfare. In Figure 13, hence, we decompose the bottom panel of Figure 11 by splitting subjects by their precision level acquired in T1. The top panel shows the kernel density estimation of welfare loss for subjects with signal precision $>1$, while the bottom panel shows the density for subjects witth precision $\leq 1 .{ }^{32}$ For both panels, solid red lines indicate the welfare density in T4, whereas dashed blue lines show the corresponding density in T1.

As for the overall case in Figure 11, the differences in the estimated distributions for the two treatments appear quite small for the two subgrups as well. We corroborate this visual intuition by formally test for the equivalence of the distributions with two-sample KS tests.

\footnotetext{
${ }^{30}$ We chose precision 1 as cut-off point because the central banks signal conveys information on whether the interest rate is in the top or bottom half of its support, which, effectively, is equivalent to a signal of precision 1 with $90 \%$ probability.

${ }^{31}$ These changes in information gathering could also simply be due to some statistical noise. However, evidence on the potential for forward guidance to increase private sector's uncertainty in the U.S. and crosscountry have been recently documented by, inter alias, Ehrmann et al. (2019).

${ }^{32}$ The numbers of subjects in the two groups are 30 for precision $>1$ and 21 for the precision $\leq 1$ respectively.
} 

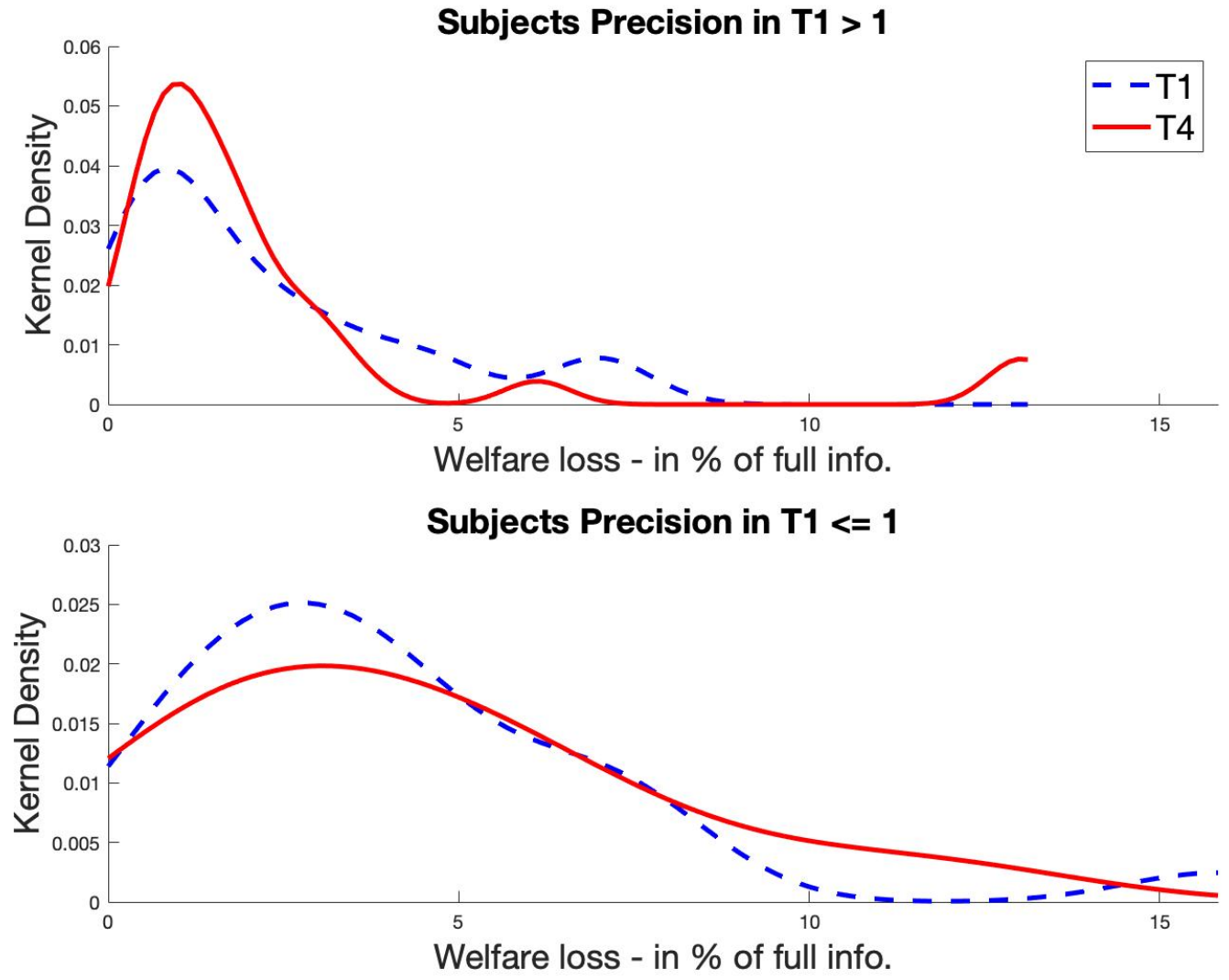

Figure 13: Kernel density estimate of welfare loss as percentage of full information for subjects who chose precision $>1$ in T1 (top panel) and subjects who chose precision $\leq 1$ in T1 (bottom panel). Red-solid lines are for T4, while blue-dashed line are for T1. The aggregate densities are estimated using a Gaussian kernel. 
The KS test shows that the null hypothesis of equality between T1's and T4's distributions cannot be rejected at level of confidence of $76 \%$ for subjects whose precision in $\mathrm{T} 1$ is $>1$ and $.99 \%$ for for those with $\mathrm{T} 1$ precision $\leq 1$ respectively.

Next, we test whether the distributions retain the same variance between the two treatments for each group to make sure that the change in information acquisition, albeit marginal, have not affected the spread of the distributions. To account for non-Gaussianity of the underlying generating process of the data, we use the Levene's test. The null hypothesis of the test is that the population variances are homoscedastic; the alternative is that they are heteroscedastic. We cannot reject the hypothesis for either group (p-values are .71 for the precision $>1$ group and .81 for the precision $\leq 1$ one respectively).

Finally, we employ the non-parametric Wilconox's signed-rank test to compare the medians of the distributions. The null hypothesis of this test is that the difference between the paired observations of the T1 and T4 samples for each subgroup follows a symmetric distribution around zero. Once again, we cannot reject the null hypothesis of equality of the mediana at very high levels of confidence (p-values of .69 for the first group and .66 for the second respectively). ${ }^{33}$

Both graphical inspection and quantitative results are in agreement in concluding that the welfare gains of going from T1 to T4 are statistically negligible. The stark monetary policy implication of this treatment is that the Odyssean forward guidance is not effective in affecting behavior and reducing welfare losses with an economy populated by rationally inattentive agents.

\subsection{Deterioration of outlook and consumption outcomes}

From the optimal theoretical solution in equation (7), dimming economic prospects captured by an increase in impatience of the private sector - a drop in $\beta$ - makes investment a less appealing option for the agents than consuming right away. This change in private sector sentiment may occur, for instance, after a prolonged expansion when consumers and businesses sense a slowdown in economic activity. In this case, the model implies that agents will increase period 1 consumption relative to the baseline case, while keeping a similar watch on movements of the interest rate.

We implement this scenario by lowering the discount factor in the computation of the lifetime utility payoff as explained in Section 3.4. Figure 14 compares the representative

\footnotetext{
${ }^{33}$ The more commonly used $F$ - and $t$ - tests for equality of variance and mean of two samples strongly rely on the assumptions of Gaussianity of the data, which are void in our case.
} 

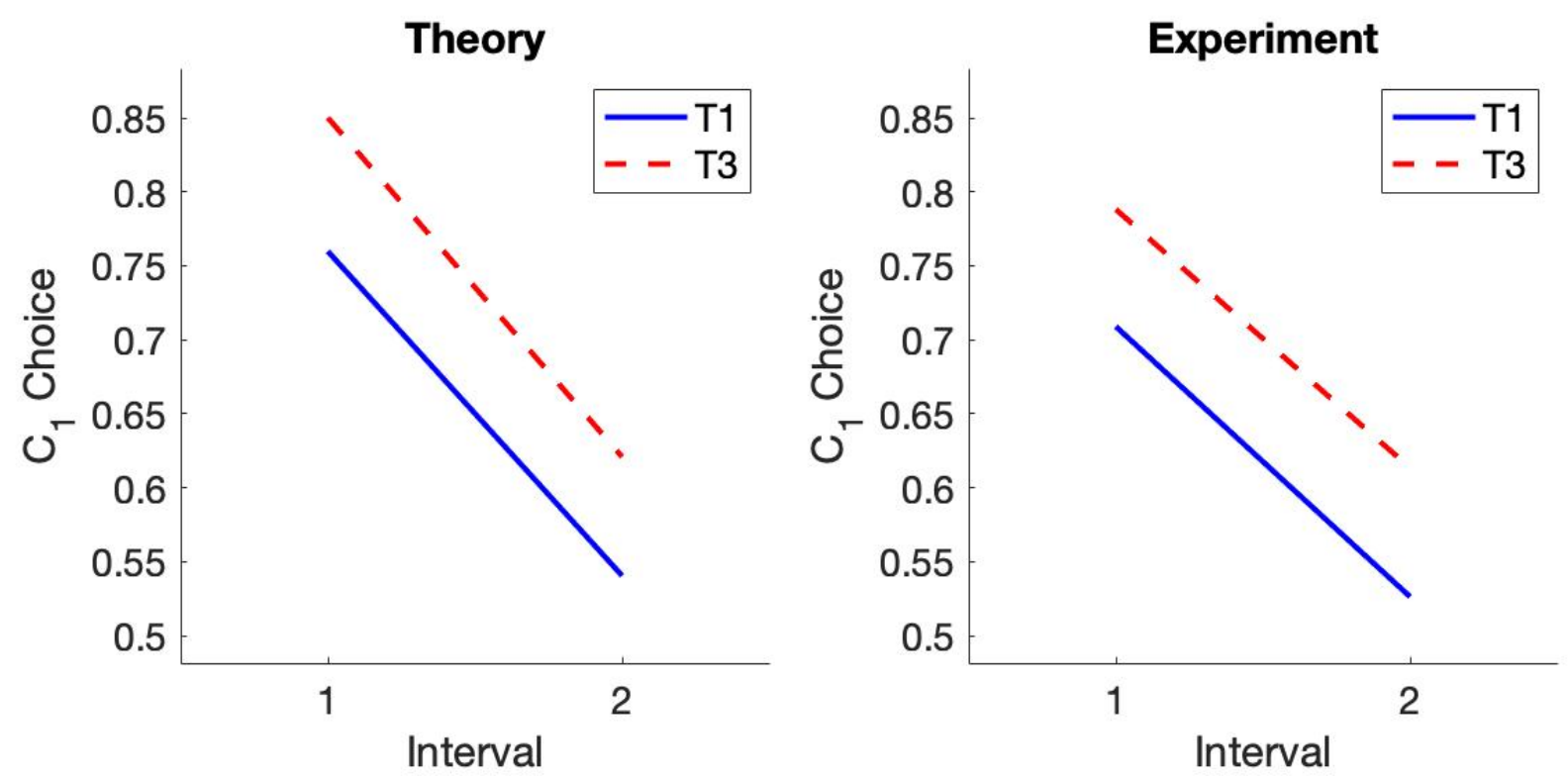

Figure 14: Comparison between the outlook deterioration treatment (T3) and the baseline (T1) consumption choices for the first period of the model $(c 1)$ made by the theoretical representative agent (left-panel) and by the subjects of the experiment, on average (rightpanel), when signal precision 1 is selected. Signal precision 1 identifies a partition of the $r$ space in two intervals of 16 columns each, as exemplified in Figure 1 . Intervals 1 and 2 then correspond to the left-hand side and right-hand side of the partition of the payoff space respectively.

agent's choice of consumption as predicted by the theory with the corresponding average choice across subjects in the experiment. We report the data for signal precision 1, which identifies a partition of the $r$ space into two 16-element intervals.

The figure illustrates a remarkable agreement between theory and experiment on the prediction that a deterioration of the outlook is associated with an increase in precautionary consumption with respect to risky investment from both the theoretical representative agent and the average participant. Moreover, as predicted by the theory, participants worried for future prospects still pay attention to the economic conditions and, specifically, to the interest rate. As Figure 6 shows, optimal signal precision for this treatment is at least as high as that for the baseline, on average. 


\section{Concluding Remarks}

This paper presents a model in which a rationally inattentive representative agent chooses consumption and savings under uncertainty about the interest rate applied to savings. A central bank can use monetary policy to persuade agents to change consumption behavior in response to changes in the stochastic properties of the interest rate. In particular, we consider two changes in policy that relate to forward guidance: a reduction in the volatility of the interest rate (a form of Delphic forward guidance) and a commitment to provide an accurate signal on the monetary policy stance (a form of Odyssean forward guidance). The model predicts that the rationally inattentive agent is not responsive to Odyssean forward guidance, while Delphic forward guidance offers her enough incentive to modify her consumption behavior. The model also predict that a deterioration of the outlook from the agent's perspective that may come from fiscal rather than monetary policy is extremely effective in inducing a change in behavior of the rationally inattentive agent, resulting in lower investment in the future. These theoretical findings are tested in a laboratory experiment. We find that subjects's choices are consistent with those predicted by the rational inattention model. Moreover, we show that the predictions of the rational inattention model are more accurate than those from the full information model in replicating subjects' behavior, thereby reinforcing the idea that people are better described as limited in their cognitive ability to process information as opposite to having infinite resources to devote to changes in monetary policy and swift and precise behavioral reactions to those changes. The experimental and theoretical results are corroborated by the recent and growing empirical literature on households' reactions to fiscal and monetary policy. To our knowledge, this is the first paper that test in the laboratory the effectiveness of the two forms of forward guidance on individual behaviors as well as changes in the outlook on consumption and savings behaviors. The paper has three starks policy implications. First, people react to changes in policy only insofar as those changes represent significant shifts of their utility. Changes that imply small deviations from the current conditions are not considered worthy of attention or behavioral responses. Second, a central bank concerned about people's welfare is best served by a policy that reduces uncertainty about the interest rate and, as a result, the economic environment than by a commitment to keep rates predictable. In our theory and experiment, the commitment to predictable rates, while it might change people's attention to monetary policy in general, has no effect on people's behavior with respect to an economic environment where this commitment is absent. Third, people's perception of the outlook as it emerges from material changes in the economic environment or fiscal landscape is the 
most important trigger of behavioral change. We think this last point together with the difference in attention and behavioral response to fiscal and monetary policies uncovered by the recent empirical evidence for the U.S. are promising venues for future research. 


\section{References}

Allcott, H. and Taubinsky, D. (2015). Evaluating behaviorally motivated policy: Experimental evidence from the lightbulb market. American Economic Review, 105 (8), S2051-S2538. https://doi.org/10.1257/aer.20131564

Andrade, P. and Ferroni, F. (2018). Delphic and Odyssean Monetary Policy Shocks: Evidence from the Euro Area. Working Paper Series WP-2018-12, Federal Reserve Bank of Chicago. https://doi.org/10.21033/wp-2018-12

Bachmann, R., Berg, T. O. and Sims, E. R. (2015). Inflation expectations and readiness to spend: Cross-sectional evidence. American Economic Journal: Economic Policy, 7 (1), 1-35.

Baeriswyl, R. and Cornand, C. (2010). The signaling role of policy actions. Journal of Monetary Economics, 57 (6), 682-695. https://doi.org/10.1016/j.jmoneco.2010.06.001

Binder, C. (2017). Fed speak on main street: Central bank communication and household expectations. Journal of Macroeconomics, 52 (C), 238-251. https://doi.org/10.1016/j.jmoneco.2017.05

Bowman, A. and Azzalini, A. (1997). Applied smoothing techniques for data analysis: the kernel approach with S-Plus illustrations. Oxford University Press, USA. https://isbn.nu//0198523963

Campbell, J. R., Evans, C. L., Fisher, J. D. M., Justiniano, A., Calomiris, C. W. and Woodford, M. (2012). Macroeconomic effects of Federal Reserve forward guidance. Brookings Papers on Economic Activity, pp. 1-80. https://doi.org/10.2139/ssrn.2166310

-, Ferroni, F., Fisher, J. D. M. and Melosi, L. (2019). The limits of forward guidance. (WP-2019-3). https://doi.org/10.21033/wp-2019-03

Caplin, A. and Dean, M. (2011). Revealed preference, rational inattention, and costly information acquisition. The American Economic Review, 101 (7), 2899-2922. https://doi.org/10.3386/w19876

- and - (2015). Revealed preference, rational inattention, and costly information acquisition. The American Economic Review, 105 (7), 2183-2203. https://doi.org/10.1257/aer.20140117 
Cheremukhin, A., Popova, A. and Tutino, A. (2015). A theory of discrete choice with information costs. Journal of Economic Behavior and Organization, 113, 3450. https://doi.org/10.1016/j.jebo.2015.02.022

Chetty, R., Looney, A. and Kroft, K. (2009). Salience and taxation: Theory and evidence. The American Economic Review, 99 (4), 1145-1177. https://doi.org/10.3386/w13330

Civelli, A. and Deck, C. (2018). A flexible and customizable method for assessing cognitive abilities. Review of Behavioral Economics, 5, 123-147. https://doi.org/10.1561/105.00000081

-, - , Tutino, A. and LeBlanc, J. (2018). Rationally inattentive consumer: An experiment. Mimeo, University of Arkansas. https://doi.org/10.24149/wp1813

Coibion, O., Gorodnichenko, Y. and Ropele, T. (2019). Inflation Expectations and Firm Decisions: New Causal Evidence*. The Quarterly Journal of Economics, qjz029. https://doi.org/10.1093/qje/qjz029

Dean, M. and Neligh, N. (2017). Experimental test of rational inattention. Mimeo, Columbia University. https://doi.org/10.7916/d8-4w4k-3q85

Easaw, J., Golinelli, R. and Malgarini, M. (2013). What determines household inflation expectations? Theory and evidence from a household survey. European Economic Review, 61, 1-13. https://doi.org/10.1016/j.euroecorev.2013.02.009

Eggertsson, G. and Woodford, M. (2003). The zero bound on interest rates and optimal monetary policy. Brookings Papers on Economic Activity, pp. 212-219. https://econpapers.repec.org/RePEc:bin:bpeajo:v:34:y:2003:i:2003-1:p:139-235

Ehrmann, M., Gaballo, G., Hoffmann, P. and Strasser, G. (2019) Can more public information raise uncertainty? The international evidence on forward guidance. Journal of Monetary Economics, 108, 93112. https://doi.org/10.1016/j.jmoneco.2019.08.012 
Eusepi, S. and Preston, B. (2018). The science of monetary policy: An imperfect knowledge perspective. Journal of Economic Literature, 56 (1), 3-59. https://doi.org/10.1257/jel.20160889

Gabaix, X., Laibson, D., Moloche, G. and Weinberg, S. (2006). Costly information acquisition: Experimental analysis of a boundedly rational model. The American Economic Review, 96 (4), 1043-1068. https://doi.org/10.1257/aer.96.4.1043

Gaballo, G. (2016). Rational inattention to news: The perils of forward guidance. American Economic Journal: Macroeconomics, 8 (1), 42-97. https://doi.org/10.1257/mac.20130337

Goecke, H., J. Luhan, W. and Roos, M. W. (2013). Rational inattentiveness in a forecasting experiment. Journal of Economic Behavior and Organization, 94, 8089. https://doi.org/10.1016/j.jebo.2013.08.013

Khaw, M., Stevens, L. and Woodford, M. (2017). Discrete adjustment to a changing environment: Experimental evidence. Journal of Monetary Economics, 91, 88-103. https://doi.org/10.1016/j.jmoneco.2017.09.001

Khaw, M. W., Stevens, L. and Woodford, M. (2016). Discrete adjustment to a changing environment: Experimental evidence. Technical report, National Bureau of Economic Research. https://doi.org/10.3386/w22978

Kumar, S., Afrouzi, H., Coibion, O. and Gorodnichenko, Y. (2015). Inflation Targeting Does Not Anchor Inflation Expectations: Evidence from Firms in New Zealand. NBER Working Papers 21814, National Bureau of Economic Research, Inc. https://doi.org/10.3386/w21814

McKay, A., Nakamura, E. and Steinsson, J. (2016). The power of forward guidance revisited. American Economic Review, 106 (10), 3133-58. https://doi.org/10.1257/aer.20150063

Melosi, L. (2016). Signalling effects of monetary policy. The Review of Economic Studies, 84 (2), 853-884. https://doi.org/10.1093/restud/rdw050

Pinkofskiy, M. (2009). Rational inattention and choice under risk: Explaining violations of expected utility through a shannon entropy formulation of the costs of $\begin{array}{lllll}\text { rationality. Atlantic Economic Journal, } 37 & \text { (1), 99-112. }\end{array}$ 


\section{https://doi.org/10.1007/s11293-008-9159-1}

Roth, C. and Wohlfart, J. (2019). How do expectations about the macroeconomy affect personal expectations and behavior? The Review of Economics and Statistics - Forthcoming. https://doi.org/10.1162/rest a 00867

Santos, B. D. L., Hortasu, A. and Wildenbeest, M. (2012). Testing models of consumer search using data on web browsing and purchasing behavior. American Economic Review, 102 (6), 2955-2980. https://doi.org/10.1257/aer.102.6.2955

Sims, C. A. (2003). Implications of rational inattention. Journal of Monetary Economics, 50 (3), 665-690. https://doi.org/10.1016/s0304-3932(03)00029-1

- (2006). Rational inattention: A research agenda. American Economic Review: Papers and Proceedings, 96, 158-163. https://econpapers.repec.org/RePEc:zbw:bubdp1:4228

Taylor, J. B. (1993). Discretion versus policy rules in practice. CarnegieRochester Conference Series on Public Policy, 39, 195 - 214. https://doi.org/10.1016/0167-2231(93)90009-L

Tutino, A. (2013). Rationally inattentive consumption choices. Review of Economic Dynamics, 16 (3), 421-439. https://doi.org/10.1016/j.red.2012.03.003

Yagan, D. (2019). Employment hysteresis from the great recession. Journal of Political Economy, 127 (5), 2505-2558. https://doi.org/10.1086/701809 
Appendix 


\section{A Instructions for the Laboratory Experiment}

After you read these instructions you will go through a 10 minute practice phase. The practice phase will not impact your payoff at all. It is designed to help you understand the choices you are making before you begin the paid phases of the experiment. After the practice phase you will have a chance to go back through the directions before beginning the paid phases.

If you have a question at any point, please raise your hand, but ...

How you use your time is an important factor in how much money you can earn, so it is best to ask any questions while the instructions are on your screen because the experiment cannot be paused during the active phases of it.

\section{Phases of the Study}

You will complete 4 paid phases that each last about 10 minutes. Each phase is a little different and you will read specific instructions before completing each phase. What you do in one phase will have no bearing on what happens in another phase. The amount you will be paid is based on the sum of what you earn in each phase. Monetary amounts in the study are denoted in Lab Dollars which are converted into \$US at the rate 100 Lab Dollars = \$US 1.

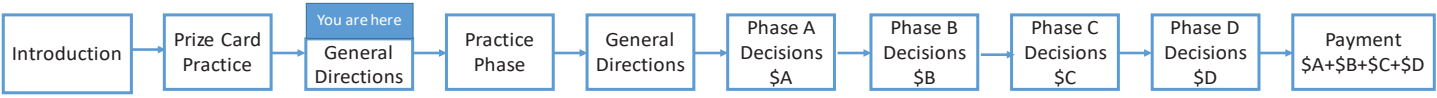

\section{Periods and Payoffs in a Phase}

Each paid phase is comprised of a series of periods. In each and every period you will pick a prize card and earn money. Prize cards work exactly like the ones in the practice you just went through.

Your prize each period will be added to the running total for that phase and a new period will begin automatically once your prize is revealed for the previous period.

There is a limit to the number of periods you can complete in a phase, but you do not know what that limit is and it can differ phase to phase. The more periods you complete, the more opportunities you have to earn money so you do not want to waste time during a phase.

NOTE: The only way you earn money is selecting a prize card (clicking on a row heading) and then confirming your choice.

The prize cards do not change period to period within a phase, but may be different in different phases. 


\section{Hints about what Ball is Drawn}

The prize ball is actually drawn before you choose your prize card. But the draw is not revealed to you until after you pick a prize card. However, you can get a hint about the ball that has been drawn.

To get a hint you have to correctly solve logic puzzles. The harder the puzzles you solve, the more accurate the hint you will receive. How logic puzzles work is explained below.

You can narrow the range of balls and thus possible prize amounts to a group of 16 by solving easy puzzles. In this case you would be informed either that the ball drawn is numbered between 1 and 16 or that the ball drawn is numbered between 17 and 32 . That is you are told if it is the first group of 16 or the second group of 16 . The way the hint is displayed on your screen is that prizes associated with balls in the group that does not contain the drawn ball are grayed out on every prize card. Prizes associated with balls in the group that does contain the ball that was drawn are highlighted in yellow on every prize card.

You can narrow the size of the group down to 8 balls (balls 1-8, balls 9-16, balls 17-24, or balls 25-32) by solving slightly harder puzzles, a group of 4 balls by moderately harder puzzles, a group of 2 balls by solving very hard problems, or even to a single ball by solving really hard puzzles.

As you will see, the puzzles can be time consuming. So you face a tradeoff of

getting a more specific hint so you can increase what you expect to earn the current period \&

getting a more vague hint and completing more periods

Returning to the previous example with only 5 prize cards and 8 balls, if you got the hint that the range for the prize ball that was drawn was between 1 and 4 then your screen would look like this:

\begin{tabular}{l|l|l|l|l|l|l|l|l|l|l|l|}
\multicolumn{10}{c|}{} & \multicolumn{10}{c|}{ Possible Prizes in Yellow } \\
& 1 & 2 & 3 & 4 & 5 & 6 & 7 & 8 \\
\hline \multirow{4}{*}{ Prize } & 1 & 2 & 3 & 4 & 5 & 6 & 7 & \\
\hline Cards & 2 & 2 & 3 & 4 & 5 & 6 & & & 6 \\
\hline & 3 & 4 & 5 & 6 & 7 & & 6 & 5 & 4 \\
\hline & 4 & 6 & 8 & 7 & 6 & 5 & 4 & 3 & 2 \\
\hline & 5 & 8 & 7 & 6 & 5 & 4 & 3 & 2 & 1 \\
\hline
\end{tabular}


Notice that the prizes for balls 5 through 8 are grayed out. This is how you know that the ball that has been drawn is not in that range. If you were to pick card 2 your prize is equally likely to be $2,3,4$, or 5 . If you were to pick card 4 , your prize is equally likely to be $6,8,7$, or 6 . Notice that card 4 has a higher prize than card 2 for all four of the balls that could be drawn so in this case picking card 4 would always earn you more money than card 2. But card 5 would earn more than card 4 if ball 1 was drawn. In general, the more accurate your hint, the better able you are to pick the card that will pay you the most money that period. But getting more accurate hints takes time and means that you can complete fewer periods

TIME PENATLY: If you do not get a hint that narrows the number of balls down to something less than 32, then you will have to wait 60 seconds before starting the next period. Keep in mind that you only have about 10 minutes in each phase and that your earnings are the sum of the prizes you earn and you only earn one prize each period.

MULTIPLE HINTS: If you successfully get a hint, you can then try to get an even more accurate hint. But you do not have to have to work your way up to a more accurate hint. That is, if you want to narrow the range down to 4 balls, you can go straight to that level rather than first narrowing it down to 16 then 8 then 4. Generally, it is better to decide how accurate of a hint you want and go straight to that option, keeping in mind that more accurate hints are harder to achieve.

FAILURE to get HINT: If you have not successfully gotten a hint in the period, it is as if you are just starting the period. You can make a choice without getting a hint, but you would incur the 60 second penalty or you can try to get a hint. If you have already successfully received a hint during the period, but failed to get a more accurate hint, it is as if you just receive the hint you already had. You can either pick a prize card or try to get a more accurate hint.

\section{Logic Puzzles}

The logic puzzles involve a 3-by-3 table of images, with the image that belongs in the lower right missing. To solve the logic puzzle you have to identify the image that completes the table from the multiple choice options provided. 
The images have six characteristics: shape, size, color, orientation, border style, and border

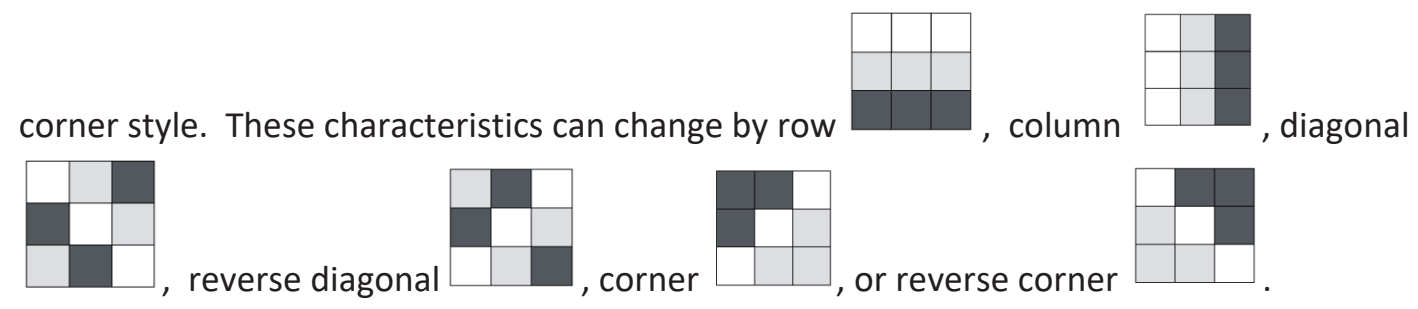

Below is an example of a logic puzzle.
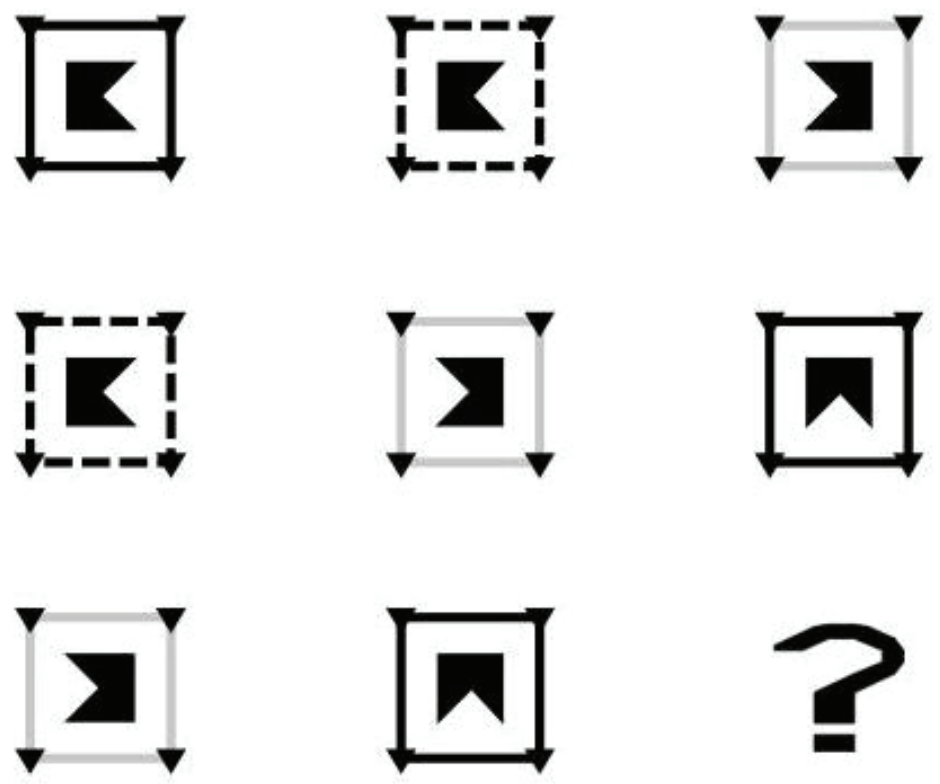

In this example, the shape is the same in each image as is the color, size, and border corner. But the border changes along the reverse diagonal. The orientation changes with the corner the image is turned in the same direction for everything in the top left (above the diagonal) of the table.

Given this, the correct answer from the choices below is " $\mathrm{D}$ " because it has a dashed border and the correct orientation while all of its other characteristics match those of the other images in the table. Notice that $\mathrm{A}$ has circles for border corners. B is the wrong size - it is too large. $\mathrm{C}$ has the wrong shape. $\mathrm{E}$ is oriented the wrong way. $\mathrm{F}$ is the wrong color. $\mathrm{G}$ and $\mathrm{H}$ both have the wrong border. 

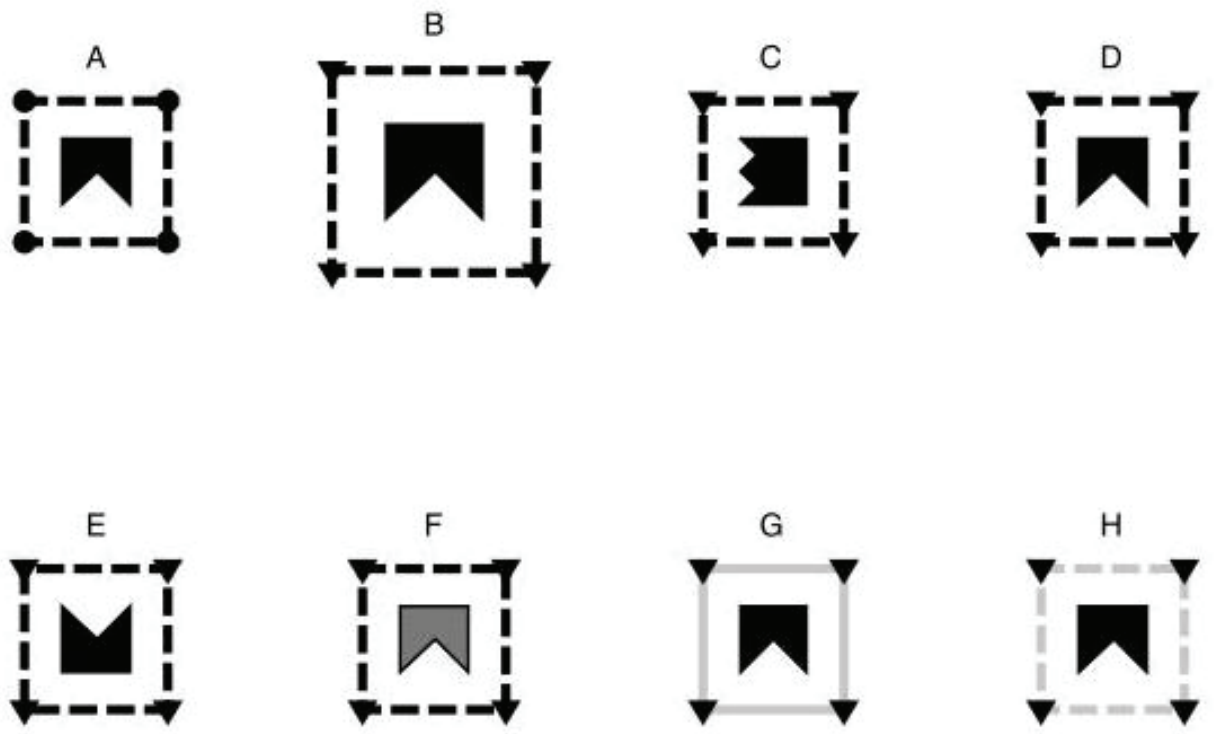

\section{Summary}

1. There are 4 paid phases that each last about 10 minutes. You are paid based on the sum of your earnings in all four phases.

2. During a period the computer will draw a prize ball numbered $1-32$, but you will not observe this draw until after you pick a prize card.

3. You will earn the prize associated with the ball drawn for the prize card you pick.

4. Before you pick a prize card, you can get a hint about what prize ball was drawn by solving logic puzzles.

a. A more accurate hint requires you to solve harder puzzles.

b. You can select an accuracy level at any point.

5. There is an unknown limit to the number of periods you can complete in a phase, but if you do not successfully get a hint that narrows the range of the drawn ball down to something less than 32 , then you will have to wait 60 seconds to start the next period.

If you have any questions, please raise your hand. Otherwise, you can click Start to go to the ten minute unpaid practice phase. After the practice phase ends you will have a chance to reread the instructions before starting the paid phases. 